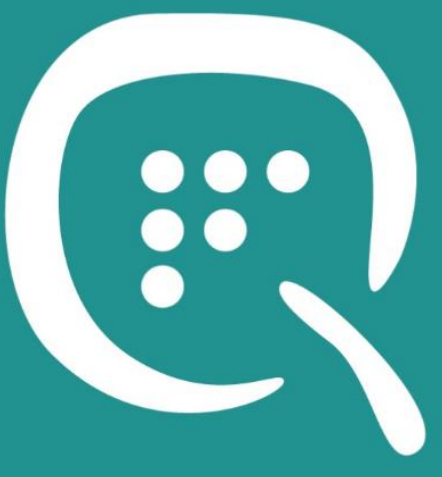

ISSN: $1909-2814$

\section{Cómo citar}

este artículo en APA:

Álvarez, G. \& Difabio de Anglat, H.

(2016). Formación virtual en

estrategias para la producción

conceptual y escrita en el posgrado

en Ciencias Sociales y Humanas.

Revista Q, 10(20), 110-136.

doi:10.18566/revistaq.v10n20.a05

Recibido: 2016-05-19

Aprobado: 2016-06-01
Formación virtual en estrategias para la producción conceptual y escrita en el posgrado en Ciencias Sociales y Humanas

\author{
Virtual Training in Strategies for Conceptual and \\ Written Production in Social and Human Sciences \\ Postgraduate Studies
}

\section{GUADALUPE ÁLVAREZ}

Doctora en Letras por la Universidad Nacional de Cuyo (Mendoza). Investigadora del Consejo Nacional de Investigaciones Científicas y Técnicas, e investigadora docente del Instituto del Desarrollo Humano de la Universidad Nacional de General Sarmiento. También participa como miembro investigador del Departamento TIC del Centro de Investigaciones en Antropología Filosófica y Cultural.

En la actualidad, estudia los modos de potenciar los usos de las nuevas tecnologías para facilitar el desarrollo de las habilidades de lectura y escritura, particularmente en ámbitos universitarios.

Email: galvarez@ungs.edu.ar

Orcid: 0000-0001-7152-730X

\section{HILDA DIFABIO DE ANGLAT}

Doctora en Ciencias de la Educación por la Universidad Nacional de Cuyo (Mendoza, Argentina). Investigadora del Consejo Nacional de Investigaciones Científicas y Técnicas, con sede en el Centro de Investigaciones Cuyo (CIC), Mendoza, el que dirige desde 2011. En la actualidad, está dedicada a investigar aspectos vinculados con la educación de nivel doctoral y, particularmente, la producción conceptual y escrita de los géneros del posgrado.

Email: ganglat@gmail.com

Orcid: 0000-0002-9679-1745

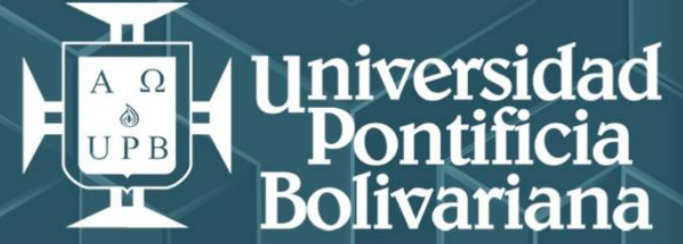

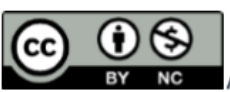

distribuir, remezclar, retocar, y crear a partir de su obra, siempre dando los créditos y sin fines comerciales. 


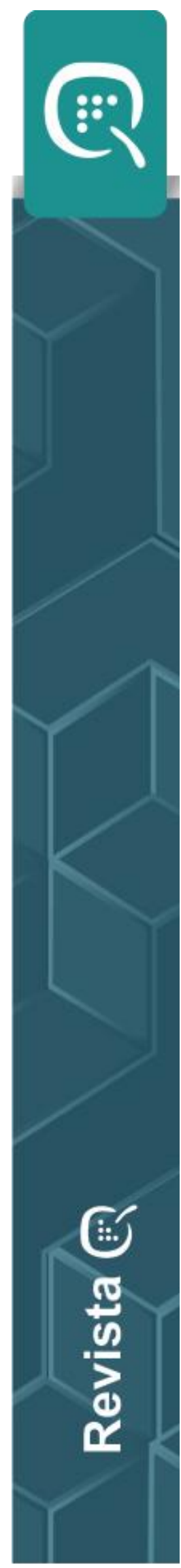

\section{Resumen}

Se lleva cabo una investigación-acción que parte del diagnóstico de dificultades de los estudiantes para la escritura académica y en el marco de la cual, de manera cíclica, se diseña, implementa, evalúa y ajusta un taller totalmente virtual mediado por tecnologías digitales (correo electrónico, blog, Googledocs) y orientado a promover la producción de un capítulo escrito por el alumno como parte de sus estudios doctorales. Este taller se desarrolla de acuerdo con una perspectiva que articula diferentes dimensiones: tecnológica, lingüísticodiscursiva, cognitiva y psico-pedagógica. En el presente artículo se comunican resultados preliminares de un primer ciclo de diseño, implementación y evaluación. En este ciclo participan once doctorandos de Ciencias Sociales y Humanas de diferentes universidades argentinas. Inicialmente, en la instancia diagnóstica, se administran cuatro instrumentos (texto cloze, inventario de escritura académica, cuestionario en torno a las concepciones de conocimiento/aprendizaje y cuestionario sobre uso de tecnologías de la escritura) y, en función de esta instancia, se diseña y se implementa una secuencia didáctica de once semanas en torno a la escritura de tesis de posgrado. Finalmente, se administran cuestionarios de evaluación de la intervención. Las apreciaciones de los estudiantes permiten deducir que la valoración general de la propuesta del taller es positiva; por otra parte, evidencian un incremento, afín con el nivel de partida, en estrategias de regulación de la escritura, la concepción de la labor escritural como elaboración, sentimientos de autoeficacia como escritor y competencia autopercibida para la escritura académica.

Palabras clave: formación virtual, estudios de posgrado, producción escritura.

\section{Abstract:}

Within an action-research project, and based on a diagnosis of student difficulties for academic writing, an entirely virtual workshop mediated by digital technologies (e- mail, blog, GoogleDocs), was cyclically designed, implemented, evaluated, adjusted and aimed to promoting the production of a chapter written by the student as part of his doctoral studies. This virtual workshop is conducted in accordance with a perspective that articulates technological, linguistic, cognitive and psycho-pedagogical dimensions. In this paper, preliminary results of the first cycle of design, implementation, and evaluation are reported. This cycle involved eleven doctoral students of Social Sciences and Humanities of different Argentine universities. Four instruments were applied in the diagnosis phase (cloze text, inventory of academic writing, questionnaire about conceptions on knowledge/learning and questionnaire about use of technologies for writing). Based on this diagnosis, a didactic sequence of eleven weeks was designed and implemented. Finally, final evaluation questionnaires were administered. The student's assessments reveal that overall assessment of the proposed workshop is positive; on the other hand, they show an increase, which is related with the starting level, in regulation strategies of writing, conception of the writing 


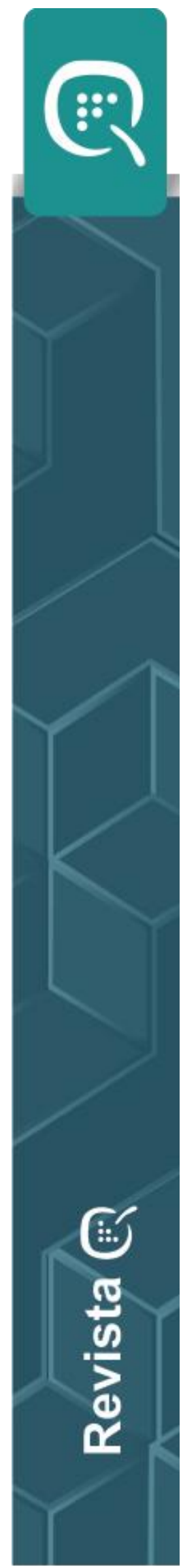

task as elaboration, feelings of self-efficacy as a writer and self-perceived academic writing competence.

Keywords: virtual teaching, postgraduate studies, written production.

\section{Datos de la investigación}

Este artículo se lleva a cabo en el marco de las investigaciones que cada una de las autoras realiza como investigadoras del Consejo Nacional de Investigaciones Científicas y Técnicas de Argentina. En particular, "La enseñanza virtual de la escritura de tesis de posgrado: las tecnologías digitales como puentes tecnoeducativos para el trabajo en grupo de pares", proyecto a cargo de Guadalupe Álvarez, y "La construcción de los géneros textuales del posgrado en Educación en entornos mixtos. Su relación con la autorregulación de la escritura y el sistema de concepciones epistemológicas", proyecto a cargo de Hilda Difabio de Anglat.

\section{Introducción}

Si bien es cada vez mayor la oferta de posgrados en las universidades públicas y privadas, y se incrementa la cantidad de alumnos de doctorados, maestrías y carreras de especialización, se registra un bajo porcentaje de estudiantes que finalmente se titula. Uno de los motivos de esta situación es la dificultad que encuentran los estudiantes para elaborar los trabajos escritos de evaluación parcial o final de las carreras (Caffarella y Barnett, 2000; Carlino, 2005). De hecho, como cuenta Carlino (2005), en los países centrales se denomina ABD (All but dissertation) a la condición de los alumnos que han cumplido todas las exigencias propias de la carrera doctoral excepto la tesis. Se ha destacado que uno de los factores que obstaculizan la producción de la tesis es que esta tarea se resuelve generalmente de modo solitario, con ausencia o escasez de orientaciones didácticas (di Stefano y Pereira, 2004).

Por otra parte, actualmente el perfil de los estudiantes doctorales es muy diverso respecto de sus antecedentes e intereses: junto con aquellos interesados en la carrera de investigación, que dedican el tiempo completo al doctorado, muchas veces ayudados por becas, se encuentran otros que realizan el doctorado a tiempo parcial, en algunos casos llevando a cabo diversos trabajos, y suelen requerir acompañamientos estratégicos y dinámicos.

Frente a este contexto, parece necesario diversificar y dar mayor continuidad a las ayudas pedagógicas que necesitan los estudiantes de posgrado (Arnoux et al., 2004). Una de las posibilidades, tal como muestra la bibliografía actual (e.g. Aitchison y Lee, 2006; Lassig et al., 2009; Maher et al., 2008), es la oferta de seminarios de tesis y/o grupos de intercambio que permitan valorar los avances de los estudiantes y que sirvan, fundamentalmente, para compartir las experiencias y comprender que las dificultades no son tan individuales.

En este sentido, resulta sumamente interesante reconocer las posibilidades que ofrecen las tecnologías digitales para potenciar de modo óptimo la interacción entre los actores y la 


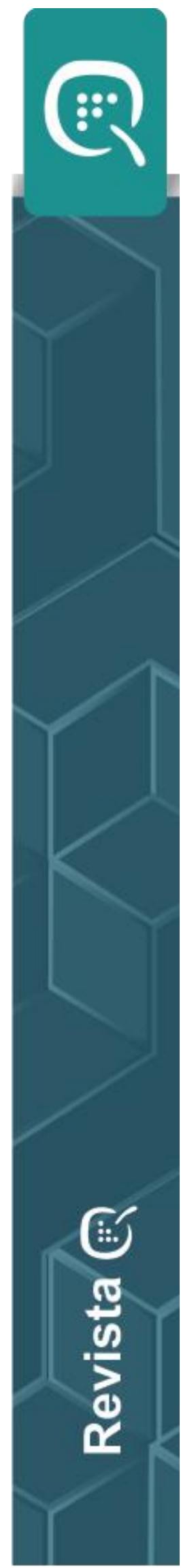

interactividad entre estos, los materiales y las actividades. Así, las propuestas educativas mediadas por tecnologías, ya sea de modo completamente virtual o semipresencial, aparecerían como modalidades muy adecuadas de acompañamiento en la formación doctoral. Sin embargo, en las bases bibliográficas (e.g. EBSCO, JSTOR, GOOGLE Académico), aun cuando el relevamiento muestra una publicación cuantiosa en torno a la redacción científica, son muy escasas las experiencias publicadas sobre acompañamiento virtual de la escritura de posgrado.

De allí que resulta relevante referirnos a una investigación-acción en el marco de la cual, de manera cíclica, se diseña, implementa, evalúa y ajusta un taller totalmente virtual mediado por tecnologías digitales (i.e., correo electrónico, blog, documentos Googledocs) y orientado a promover la producción de un capítulo escrito por el alumno como parte de sus estudios doctorales. Este taller se desarrolla de acuerdo con una perspectiva que articula diferentes dimensiones: tecnológica, lingüístico-discursiva, cognitiva y psico-pedagógica. En el presente artículo se comunican los resultados de un primer ciclo de diseño, implementación y evaluación. Este ciclo ha sido desarrollado en el marco de la Facultad de Filosofía y Letras de la Universidad Nacional de Cuyo con el objeto de orientar la escritura de la tesis de posgrado de Ciencias Sociales y Humanas. El propósito de este artículo es, entonces, presentar los fundamentos pedagógicos, didácticos y tecnológicos de dicha propuesta, así como algunos resultados -aún preliminares y muy parciales- relativos tanto a los cuestionarios del diagnóstico como a los cuestionarios realizados al final de la intervención.

\section{Fundamentos pedagógicos, didácticos y tecnológicos de la propuesta}

Esta propuesta fue diseñada en función de una serie de fundamentos pedagógicos, didácticos y tecnológicos específicos.

En primer lugar, concebimos la escritura como un instrumento clave para el trabajo intelectual y el desarrollo de operaciones cognitivas de orden superior (Bereiter y Scardamalia, 1987; Flower, 1979; Flower y Hayes, 1996). Esto se debe, por un lado, al carácter fijo y permanente de la palabra escrita, que permite al lector-escritor distanciarse del texto para revisarlo y volver sobre él, es decir, permite "objetivar" el pensamiento y convertirlo en materia de reflexión. Por otro lado, por tratarse de un tipo de comunicación diferida, la escritura ofrece la posibilidad de realizar una serie de operaciones de planificación y de revisión, a partir de las cuales se puede reformular total o parcialmente el escrito.

En segundo lugar, y en relación estrecha con el supuesto anterior, consideramos que la escritura académica, tanto de grado como de posgrado (e.g. los trabajos finales de seminarios, los proyectos de investigación y las tesis), exige que el escritor sea capaz de resolver no solo los aspectos relativos al contenido, sino también los aspectos retóricos, para lo cual se requiere, junto al dominio de las temáticas, un conocimiento de los géneros específicos que circulan en el nivel académico y de las características propias de sus dispositivos enunciativos (di Stefano y Pereira, 2004). De acuerdo con ello, hemos propuesto la reconstrucción, entre otras dimensiones, del Modelo de situación e interacción comunicativa, el Modelo de evento 


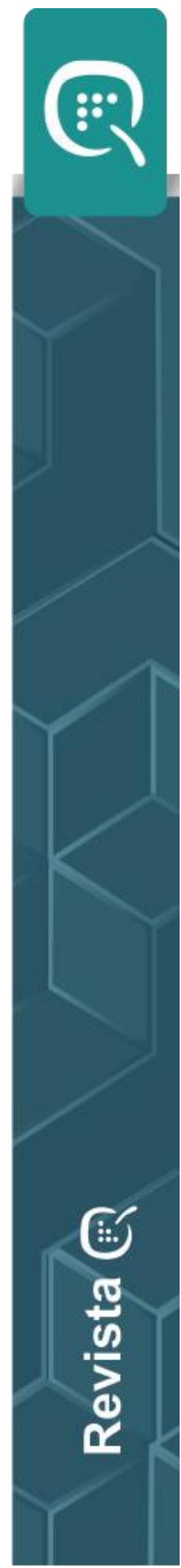

y el Modelo textual subyacentes (Cubo de Severino, Lacon y Puitti, 2011, pp. 29-30). El Modelo de situación comunicativa refiere a las relaciones sociales y los roles comunicativos de los participantes en la interacción y su contexto. El Modelo de evento da cuenta del proceso de investigación, el contenido y la realidad extralingüística a la que se refiere la tesis. El Modelo textual se vincula con las características que asume el texto de acuerdo con las tradiciones discursivas, las instancias y factores de la comunicación lingüística y sus posibilidades de variación. En particular, abarca los recursos y estrategias utilizados para expresar por escrito los significados que se quieren comunicar. De acuerdo con estos modelos, la propuesta ha buscado facilitar a los estudiantes la identificación del contexto y la situación comunicativa de una tesis, del significado y contenido de la investigación, de la estructura canónica del texto. Por otra parte, se ha entendido que las reconstrucciones de esos modelos, realizadas durante los procesos de comprensión, contribuirían con el desarrollo de los procesos de producción textual, y a la inversa (Parodi, 1999, 2001). Esto es, se ha esperado que dicho desarrollo y retroalimentación se constituyeran en un espacio propicio para que el alumno logre iniciar el tránsito desde cursante de seminarios, esto es, un consumidor del conocimiento “(...) cuidadosamente repartido en cursos, módulos, esquemas y listas de lectura, temas de conferencia y tareas de evaluación constreñidas y controladas" (Lovitts, 2005, p. 138) a "enunciador autorizado de cara a la comunidad científica de futuros pares" (Carlino, 2005, p. 52).

En tercer lugar, los procesos implicados en la producción escrita, instancias recursivas para las distintas partes de la tesis (planificación, puesta en texto y revisión), han sido concebidos como estrategias de autorregulación; esto es, desde la perspectiva psicopedagógica, se trabaja la escritura autorregulada (Zimmerman y Risemberg, 1997; Difabio de Anglat, 2012) en relación con el ambiente físico y social en el que se escribe, los aspectos personales y conductuales. En este sentido, se busca andamiar y promover el monitoreo deliberado del propio desempeño, que asegura el mantenimiento y realización de las acciones planeadas (implica que el escritor esté consciente de sus objetivos, desarrolle una representación personal de la tarea que se ajuste a la situación comunicativa y que maneje los aspectos cognitivos y afectivos durante el proceso de composición), la regulación del esfuerzo -que protege el logro de la meta frente a las dificultades o distracciones durante el transcurso de la acción-, la búsqueda de asistencia y apoyo del grupo, la estructuración del ambiente y del tiempo para controlar las condiciones de escritura.

En cuarto lugar, se postula la incidencia de las concepciones (de conocimiento/aprendizaje, de investigación y de escritura) en el proceso investigativo y en la producción conceptuallingüística, entendiendo por tales redes de creencias, ideas, supuestos, con la credibilidad suficiente para guiar las acciones. Esto es, se extiende el constructo concepciones para constituir lo que se puede denominar un concepto-sistema, una epistemología personal según Hofer (2001, 2004). Respecto de la escritura, afirma Hounsell (1987, citado por Campbell, Smith y Brooker, 1998, p. 459): “Comprender qué constituye el discurso académico representa el tipo de revolución intelectual personal demarcada en el esquema del desarrollo de Perry"1, esquema que postula el tránsito desde una visión monolítica (el conocimiento como no 


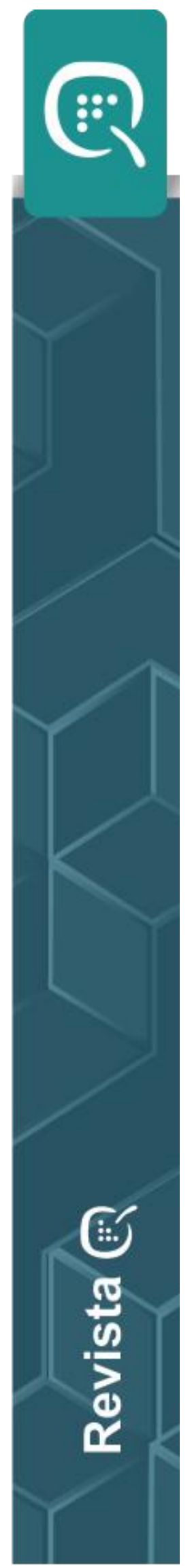

problemático y que no necesita ser justificado) a una visión evaluadora del conocimiento y su formulación.

Finalmente, hemos considerado una conceptualización específica acerca del rol de las tecnologías digitales en la escritura, particularmente cuando esta actividad se lleva a cabo entre pares. Para ello, hemos partido de los importantes beneficios que las tecnologías digitales han demostrado en otros niveles educativos para la revisión y escritura entre pares (también llamada escritura cooperativa o colaborativa) (e.g. Álvarez y Bassa, 2013; Davioli, Monari y Severinson, 2009; Passig y Swartz, 2007). Dado que hemos propuesto el andamiaje de la escritura mediado por tecnologías desde una perspectiva autorreguladora, hemos advertido que en entornos abiertos se deben tener en cuenta el todo colectivo y situado, la relación de los actores entre sí y con las herramientas y el modo en que ello constituye un sistema que estructura las actividades y la autorregulación, en tanto -como señala Cassany (2000)- se manejan diversidad de canales y códigos comunicativos, se propicia la interacción simultánea y la trasmisión instantánea, el perfil cognitivo del escritor.

\section{Metodología}

Desde el punto de vista metodológico, este estudio asume el diseño de una investigaciónacción que construye el conocimiento por medio de la práctica (Hernández Sampieri, Fernández Collado y Baptista Lucio, 2010). Se trata de un tipo de diseño que parte de problemas concretos y situados, y busca intervenir en las problemáticas diagnosticadas para transformarlas. Para ello, se desarrollan ciclos sucesivos de diagnóstico, diseño e implementación, evaluación y retroalimentación.

En el presente artículo, como hemos adelantado, nos referimos a una investigación-acción en el marco de la cual, de manera cíclica, se diagnostican problemas vinculados con la escritura académica y luego se diseña, implementa, evalúa y ajusta un taller totalmente virtual mediado por tecnologías digitales y orientado a promover la producción de un capítulo escrito por el alumno como parte de sus estudios doctorales. Aquí nos centramos en uno de los ciclos, en el cual participaron un grupo de 11 estudiantes de doctorado ( 9 mujeres y 2 varones) de Ciencias de la Educación y otras Ciencias Sociales y Humanas de diferentes universidades argentinas y dos investigadoras docentes. Una de las investigadoras tiene formación de grado en Ciencias de Educación y se ha especializado en la lectura y la escritura de textos académicos; la otra, con formación de grado en Letras, se ha especializado en enseñanza de la lectura y la escritura académicas, así como en educación mediada por tecnologías. En todos los ciclos de la investigación, ambas investigadoras han sido las responsables del diseño de la propuesta didáctica, así como de su implementación y evaluación.

En la instancia diagnóstica, implementamos:

Texto cloze 2 : el procedimiento cloze es una medida eficaz de la competencia lectora (Dastjerdi y Talebinezhad, 2006; Difabio de Anglat, 2008; Kletzien, 1991, entre otros). Como señala 


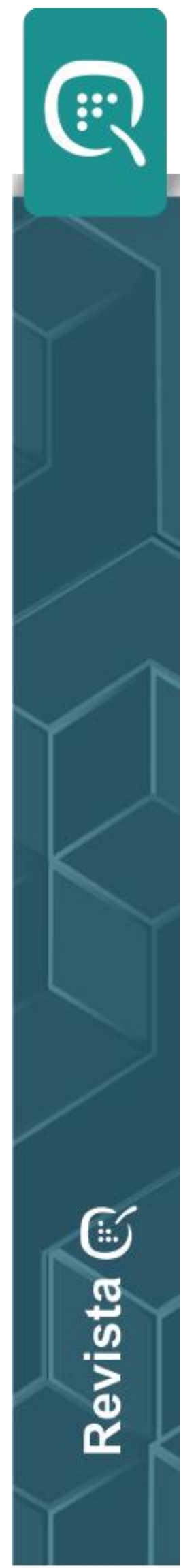

Kletzien (1991, p. 72), los procesos intelectuales implicados en la técnica (anticipación, razonamiento, evaluación, juicio, resolución de problemas) son los mismos que son esenciales para la lectura. Para esta experiencia pedagógica, se adaptó ligeramente parte del texto Principios para un final: reflexiones en torno a la escritura de la tesis (Álvarez, 2011). En su aplicación en esta muestra constreñida alcanza una confiabilidad (alpha de Cronbach) de 0,73, índice que se considera aceptable para instrumentos elaborados ad hoc (Hernández Sampieri et al., 2010). Respecto del nivel de dificultad de los ítems (que se calcula en cada uno mediante un procedimiento sencillo: porcentaje de participantes que lo resuelven correctamente -ya sea verbatim o alternativa pertinente-), según los criterios de Backhoff Escudero, Larrazolo Reyna y Rosas Morales (2000), el 64\% del cloze resulta fácil o muy fácil, el 29\% corresponde a la categoría moderado y el 7\%, a difícil o muy difícil.

Inventario de escritura académica (Difabio de Anglat, 2012): es una escala Likert tradicional en cinco opciones de respuesta, desde muy de acuerdo a muy en desacuerdo-, inicialmente de 70 ítems $^{3}$ (la mayoría, favorables) compuesta por cuatro variables, cuyas dimensiones y número de ítems se muestran en la Tabla 1:

Tabla 1. Variables y dimensiones del inventario de escritura académica $(N=110)$

\begin{tabular}{|c|c|c|c|}
\hline VARIABLES & DIMENSIONES & No DE ÍTEMS & ALPHA \\
\hline $\begin{array}{l}\text { Estrategias de escritura } \\
\text { (18 ítems) }\end{array}$ & $\begin{array}{l}\text { Planificación } \\
\text { Búsqueda de información } \\
\text { Puesta en texto } \\
\text { Revisión }\end{array}$ & $\begin{array}{l}4 \\
3 \\
5 \\
6\end{array}$ & $\begin{array}{l}0,59 \\
0,55 \\
0,67 \\
0,85\end{array}$ \\
\hline $\begin{array}{l}\text { Estrategias de regulación } \\
\text { de la escritura } \\
\text { ( } 22 \text { ítems) }\end{array}$ & $\begin{array}{l}\text { Valor de la tarea } \\
\text { Control } \\
\text { Búsqueda de asistencia } \\
\text { Apoyo del grupo } \\
\text { Estructuración del ambiente y del tiempo } \\
\text { (NO) Procastinación }{ }^{4}\end{array}$ & $\begin{array}{l}3 \\
7 \\
3 \\
2 \\
4 \\
3\end{array}$ & $\begin{array}{l}0,51 \\
0,77 \\
0,57 \\
0,36 \\
0,65 \\
0,63\end{array}$ \\
\hline $\begin{array}{l}\text { Concepciones sobre la } \\
\text { escritura (23 ítems) }\end{array}$ & $\begin{array}{l}\text { Escritura como elaboración } \\
\text { Escritura como reproducción }\end{array}$ & $\begin{array}{l}13 \\
10\end{array}$ & $\begin{array}{l}0,78 \\
0,68\end{array}$ \\
\hline $\begin{array}{l}\text { Autoeficacia para la } \\
\text { escritura (15 ítems) }\end{array}$ & $\begin{array}{l}\text { Autopercepción positiva } \\
\text { Logros de ejecución } \\
\text { (NO) Pensamientos negativos }\end{array}$ & $\begin{array}{l}4 \\
7 \\
4\end{array}$ & $\begin{array}{l}0,72 \\
0,72 \\
0,67\end{array}$ \\
\hline
\end{tabular}

Además, se obtiene una puntuación total mediante la suma de los indicadores (55 ítems) de estrategias para la escritura, para su regulación y autoeficacia (esto es, se excluyen las concepciones de este compuesto), denominada "competencia auto-percibida para la escritura académica". El alpha resulta excelente $(0,92)$.

Inventario sobre concepciones de aprendizaje/conocimiento: se aplican el cuestionario sobre Concepciones del Aprendizaje -CONAPRE- (Martínez-Fernández, 2004, 2007) y algunas dimensiones de la subescala Modelos mentales del aprendizaje del Inventory Learning Style ILS para su sigla en inglés- (Vermunt, 1994, 2005). Para su categorización, se adoptan las dimensiones del primero, las que Martínez-Fernández, en seguimiento de Pozo y Scheuer (1999), clasifica en un esquema tripartito: 1) directa, reproductiva -recibir y recordar-; el conocimiento es una copia fiel de la realidad (un saber "cerrado"), es decir que, desde el punto 


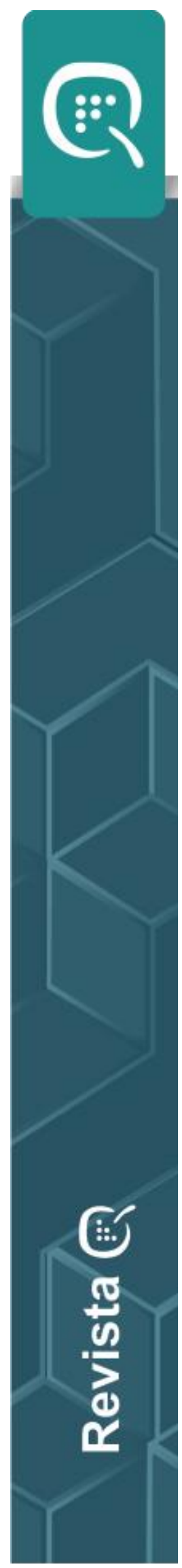

de vista epistemológico, corresponde a un realismo ingenuo; 2 ) interpretativa, se entiende el aprendizaje como producto de una actividad individual significativa, pero sin alejarse del modo de presentación del contenido, esto es, si bien la actividad del aprendiz es "el proceso crucial que media entre las condiciones y los resultados" (Martínez-Fernández, 2004, p. 86), dicho resultado se concibe como un producto único y verdadero; 3 ) constructiva, una auténtica reelaboración, cuyos procesos "posiblemente específicos a cada situación de aprendizaje (...) transforman el objeto de aprendizaje generando nuevos conocimientos que, en este caso, ya no se corresponderían con una realidad única y objetiva, accesible de modo directo" (Amezcua Prieto, Muñoz García y Amezcua Membrilla, 2012, p. 700). Se trata, entonces, de una concepción del aprendizaje en la que prima su función de "transformar el contenido y ser transformado" (Morchio y del Río Bayarri, 2015, p. 115).

A partir de la correlación corregida entre cada ítem y el puntaje total de la dimensión correspondiente, configuramos un instrumento ad hoc que combina aquellos ítems de ambas escalas que, desde la teoría y el análisis de contenido de los indicadores, muestran estrecha correspondencia, desestimando los ítems con correlaciones bajas (inferiores a 0,35):1) concepción directa conformada por seis ítems (alpha de Cronbach $=0,71$ ), 2) concepción interpretativa, también de seis ítems (alpha de Cronbach $=0,69$ ) y 3) concepción constructiva de ocho ítems (alpha de Cronbach $=0,88$ ). Las diferencias halladas en la muestra son estadísticamente significativas ( $X 2=12,047 ; p=0,002)$.

Cuestionario sobre el empleo de tecnologías digitales: se trata de una serie de preguntas en torno al uso de las tecnologías digitales e interactivas, particularmente de aquellas utilizadas para la escritura. Por un lado, se interroga por el tipo de procesador de textos empleado y el uso de sus diferentes funcionalidades. Por otro lado, se interroga sobre la frecuencia de navegación en Internet y sobre las aplicaciones utilizadas. En este sentido, se pregunta sobre las plataformas educativas utilizadas y sus funcionalidades, así como sobre las aplicaciones de la Web 2.0 que permiten archivar y compartir documentos (i.e. Dropbox) y escribir grupalmente (Googledocs). Dado el carácter del análisis que se implementa, estos datos se computan de modo descriptivo en la muestra total.

En cuanto al diseño del taller hemos establecido como objetivos, que los estudiantes:

- Analicen el modo en que el enunciador académico se construye en su discurso y diseña su auditorio, de manera que se conciban a sí mismos y a sus destinatarios como "personajes" de la escena discursiva que el escrito propone;

- comprendan cuáles son los rasgos de la tesis como género, es decir, sus regularidades discursivas y textuales;

- logren superar los problemas que plantea la puesta en discurso de la tesis a partir de la reflexión sobre la producción existente, incluyendo la propia producción;

- conozcan y apliquen correctamente las reglas gramaticales, sintácticas y semánticas del código lingüístico;

- construyan y consoliden estrategias metacognitivas que les permitan controlar conscientemente sus procesos de escritura. 


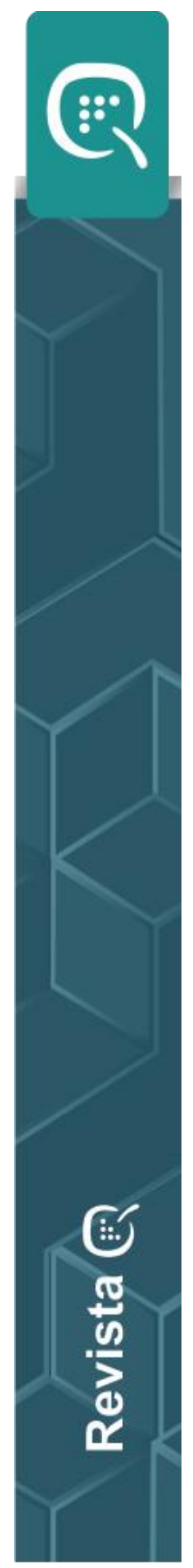

La secuencia didáctica se ha diseñado recurriendo a diversas tecnologías para cumplimentar funcionalidades específicas:

- Correo electrónico: se ha utilizado para el envío y la recepción de documentos de trabajo (bibliografía, documento con actividades). También se lo ha empleado para recibir los trabajos de los estudiantes. En este sentido, ha cumplido las funciones que en la plataforma cumplen las etiquetas y la subida de archivos.

- Blog del taller: se ha configurado como privado para los participantes del taller y ha permitido las interacciones grupales. Así, en el blog, se han planteado una o más entradas por semana ligadas a las actividades propuestas para dicha semana. Los estudiantes participaban respondiendo con comentarios a cada entrada. Por ejemplo, en una entrada del Módulo 1 se los invitaba a que consultasen las dudas relativas a la actividad propuesta para el módulo 1 y a que consultasen dudas a partir de los comentarios a esa entrada. El blog ha funcionado, entonces, del modo en que lo hacen los foros en una plataforma educativa.

- Googledocs: ha permitido la elaboración de las actividades grupales. Los estudiantes han utilizado el documento compartido, los comentarios al documento y el chat para la interacción. De esta manera, los documentos googledocs han funcionado como wikis y chat propios de una plataforma.

En suma, las diferentes funciones que ofrecen las plataformas educativas como Moodle han sido cubiertas por distintas herramientas y programas de Internet, en especial de la Web 2.0.

Por otra parte, la secuencia didáctica se diseñó según un cronograma de 11 semanas, con temas y actividades determinados en relación con cada una. La primera semana estuvo dedicada a la presentación del seminario y a las actividades de diagnóstico. La segunda semana se orientó a la reflexión sobre la escritura, en general, y la escritura académica, en particular. Las siguientes siete semanas se dedicaron al trabajo con los diferentes elementos paratextuales (título, índice resumen) y las secciones fundamentales de la tesis (introducción, marco teórico, metodología, análisis de resultados, conclusiones). Finalmente, se dedicaron dos semanas para realizar el primer borrador del trabajo final que consistió en la redacción de un capítulo o fragmento de la propia tesis de cada estudiante.

Las actividades apuntaron fundamentalmente a la búsqueda y el análisis de los elementos paratextuales y las diferentes secciones de la tesis. Este análisis comprendía la reflexión en torno al modelo de la situación comunicativa, al modelo del evento y al modelo textual. En algunos casos, se proponía realizar este tipo de ejercicio de manera individual; en otras, en pequeños grupos. Los grupos eran propuestos por los docentes en función del área de trabajo y la temática de la tesis de los estudiantes. En un caso, se propuso una actividad en la que debió intervenir el grupo completo analizando fragmentos de una sección de la tesis. En todos los casos, las docentes realizaban una devolución de las actividades realizadas. 


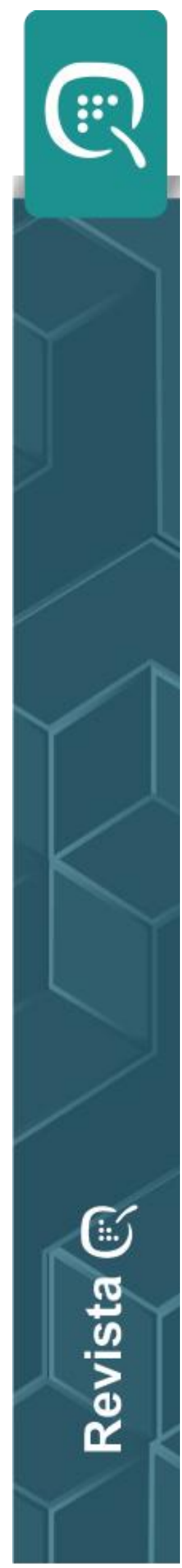

Como cierre de la propuesta, se aplicaron los siguientes instrumentos: 1) el cuestionario 1 de evaluación de las concepciones de investigación; 2) el inventario (instrumento 2) de escritura académica en el posgrado y 3 ) el cuestionario 3 con preguntas abiertas sobre la valoración de los estudiantes en torno a los aportes y limitaciones de las actividades individuales y grupales El instrumento 2 ha sido descripto previamente. El cuestionario 1 indaga sobre las concepciones de investigación mediante dos preguntas abiertas (referidas a la definición personal de investigación científica y nociones que ha modificado a partir de la experiencia de desarrollo de la tesis doctoral) y dos cerradas (sobre funciones de la investigación científica y características de la "buena" investigación). El cuestionario 3 comprende una serie de interrogantes que apuntan a relevar la perspectiva de los estudiantes sobre los aportes y limitaciones de las actividades individuales y las actividades grupales propuestas a lo largo del taller, y sobre el taller en general. Respecto de estos dos instrumentos, se implementa un análisis cualitativo de la información por medio del siguiente procedimiento en cada uno: individualmente, las dos investigadoras examinaron en detalle las respuestas y las codificaron desde categorías teóricas y emergentes de los datos; luego, consensuaron una categorización común y reexaminaron los protocolos dos o más veces en función de dicha categorización.

Finalmente, se realizó una evaluación de la intervención en base al análisis los datos obtenidos en cada una de las etapas previas. En este artículo, exponemos, de manera preliminar, los resultados relativos a los cuestionarios que han funcionado como diagnóstico, así como a los cuestionarios finales. Los análisis estadísticos que habilita el tamaño de la muestra (efectuados mediante el software SPSS 22.0) son: 1) análisis de las agrupaciones que se conforman en la interacción de las variables del diagnóstico (clúster de k medias en la función "solo clasificar"), 2) frecuencia absoluta -presentada en gráficos- acerca del empleo de tecnologías digitales, 3) comparación entre pre- y post-test en el inventario de escritura académica y ponderación de la significatividad del incremento, a través de la $U$ de Mann-Whitney en las puntuaciones medias y de $\mathrm{Chi}^{2}$ en las categorías de desempeño (baja, media y alta), 4) grado de asociación ( $V$ de Cramer) entre concepciones de investigación y de conocimiento/aprendizaje y 5) análisis de las agrupaciones que se constituyen en concepciones para un nuevo análisis de clúster respecto de las variables continuas.

\section{Análisis de los resultados}

\section{Sobre el diagnóstico}

A fin de acercarnos a un perfil diagnóstico de los cursantes, se analizan las agrupaciones que se conforman en la interacción de las variables en examen: competencia lectora, seis variables tomadas del inventario de escritura académica ${ }^{5}$ y las tres concepciones de aprendizaje/conocimiento. Los resultados, en valores medios, son: 


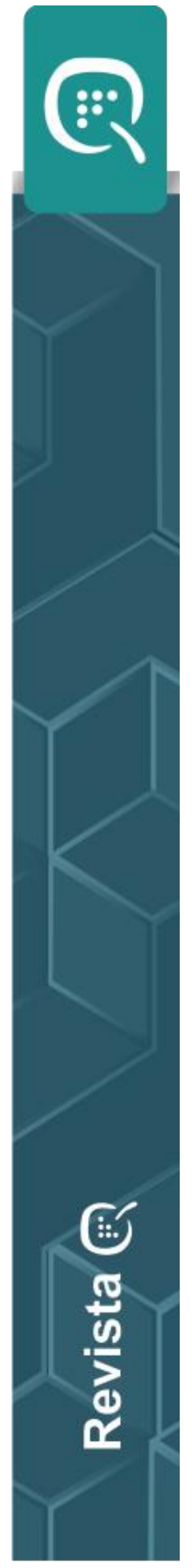

Tabla 2. Agrupaciones que se conforman en la interacción de las variables en examen

\begin{tabular}{|c|c|c|c|}
\hline \multirow{2}{*}{$\begin{array}{c}\text { Texto cloze, variables del inventario de escritura } \\
\text { académica y concepciones de } \\
\text { aprendizaje/conocimiento }\end{array}$} & \multicolumn{3}{|c|}{ Agrupación } \\
\hline & 1 & 2 & 3 \\
\hline Cloze $^{6}$ & 8,1 & 8,7 & $\overline{9,3}$ \\
\hline Estrategias de escritura & 6,27 & 7,62 & 8,85 \\
\hline Estrategias de regulación de la escritura & 5,31 & 5,96 & 7,12 \\
\hline Escritura como elaboración & 5,77 & 7,34 & 8,65 \\
\hline Escritura como reproducción & 7,50 & 5,92 & 5,50 \\
\hline Autoeficacia para la escritura & 5,27 & 5,85 & 6,88 \\
\hline Competencia autopercibida para la escritura académica & 5,37 & 6,44 & 7,58 \\
\hline Concepción directa & 6,67 & 4,24 & 5,67 \\
\hline Concepción interpretativa & 6,67 & 7,08 & 8,89 \\
\hline Concepción constructiva & 5,75 & 6,41 & 8,23 \\
\hline
\end{tabular}

La primera agrupación, en la que se ubican cuatro alumnos, se caracteriza por las puntuaciones más bajas en todas las variables, a excepción de escritura como reproducción y, en correspondencia con ello, en concepción directa.

La segunda está conformada por valores medios, nuevamente excluyendo las dos dimensiones mencionadas ya que, respecto de la anterior, disminuyen de manera sensible, sobre todo la concepción directa, que representa el puntaje inferior de la muestra. Tres estudiantes se sitúan en esta segunda agrupación.

La última corresponde al mejor desempeño y a la puntuación más baja en escritura como reproducción; subgrupo constituido por cuatro alumnos.

En su representación gráfica:

Gráfico 1. Valores medios de las variables en examen en función del desempeño

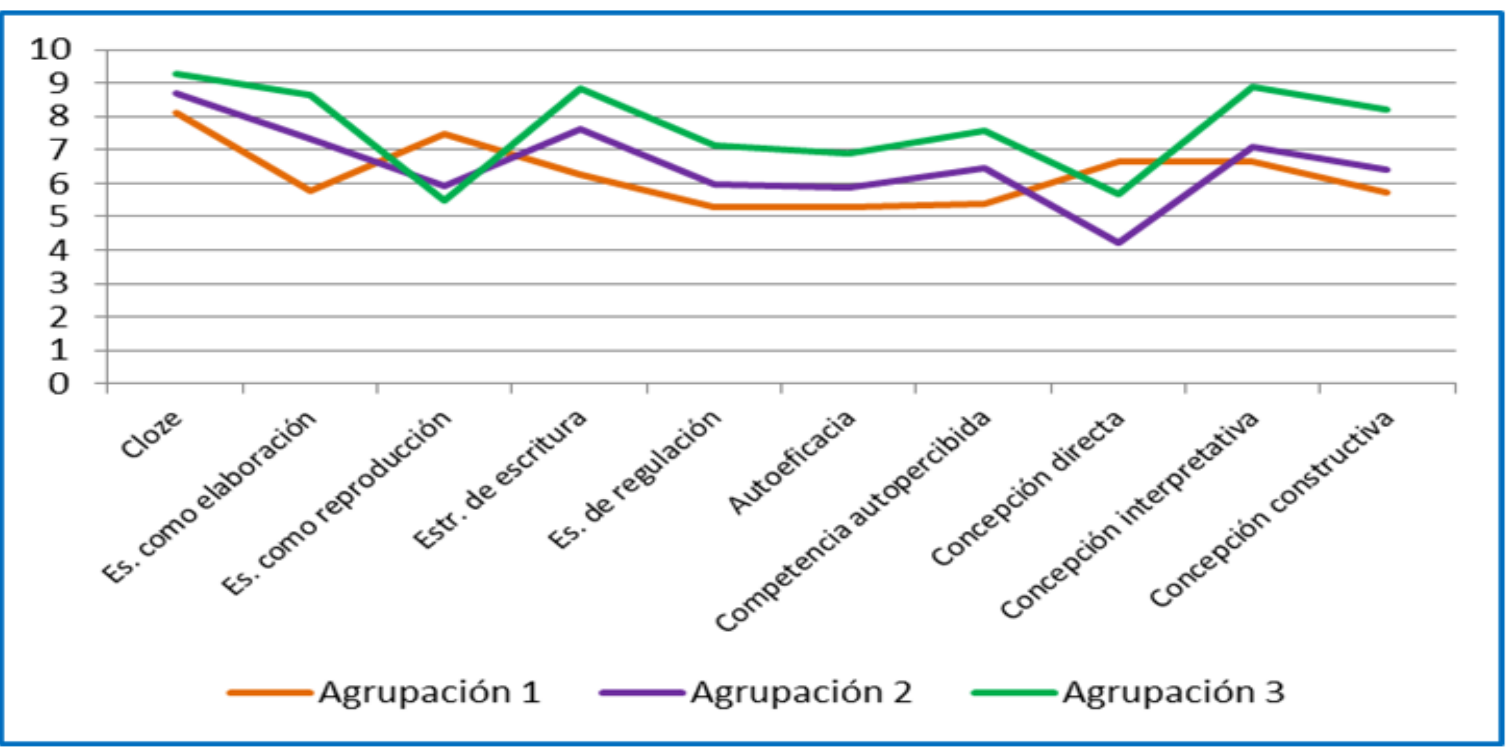

Revista Q | Vol. 10 | No. 20 | enero-junio 2016 


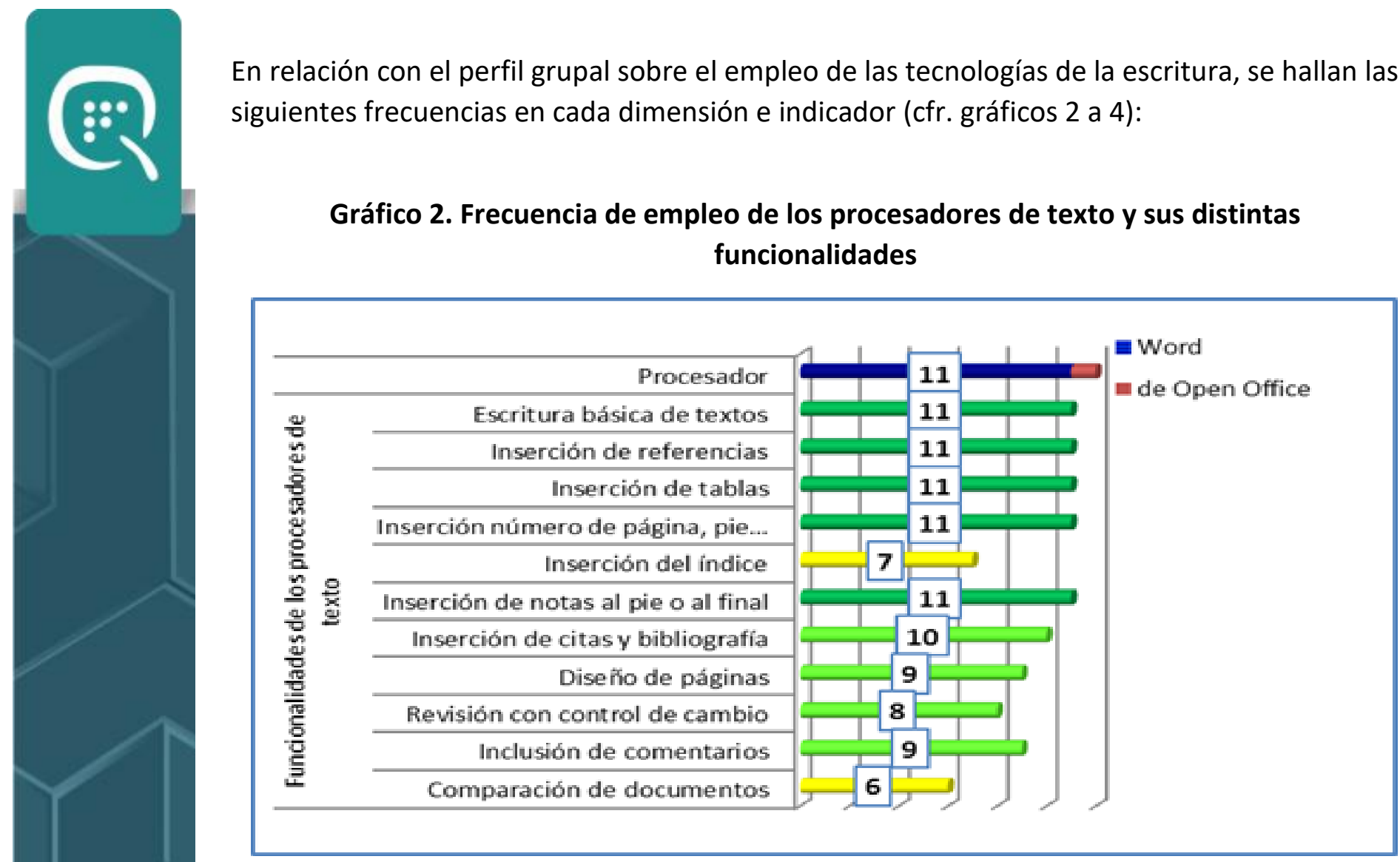

Gráfico 3. Frecuencia de uso de Internet y de las aplicaciones educativas

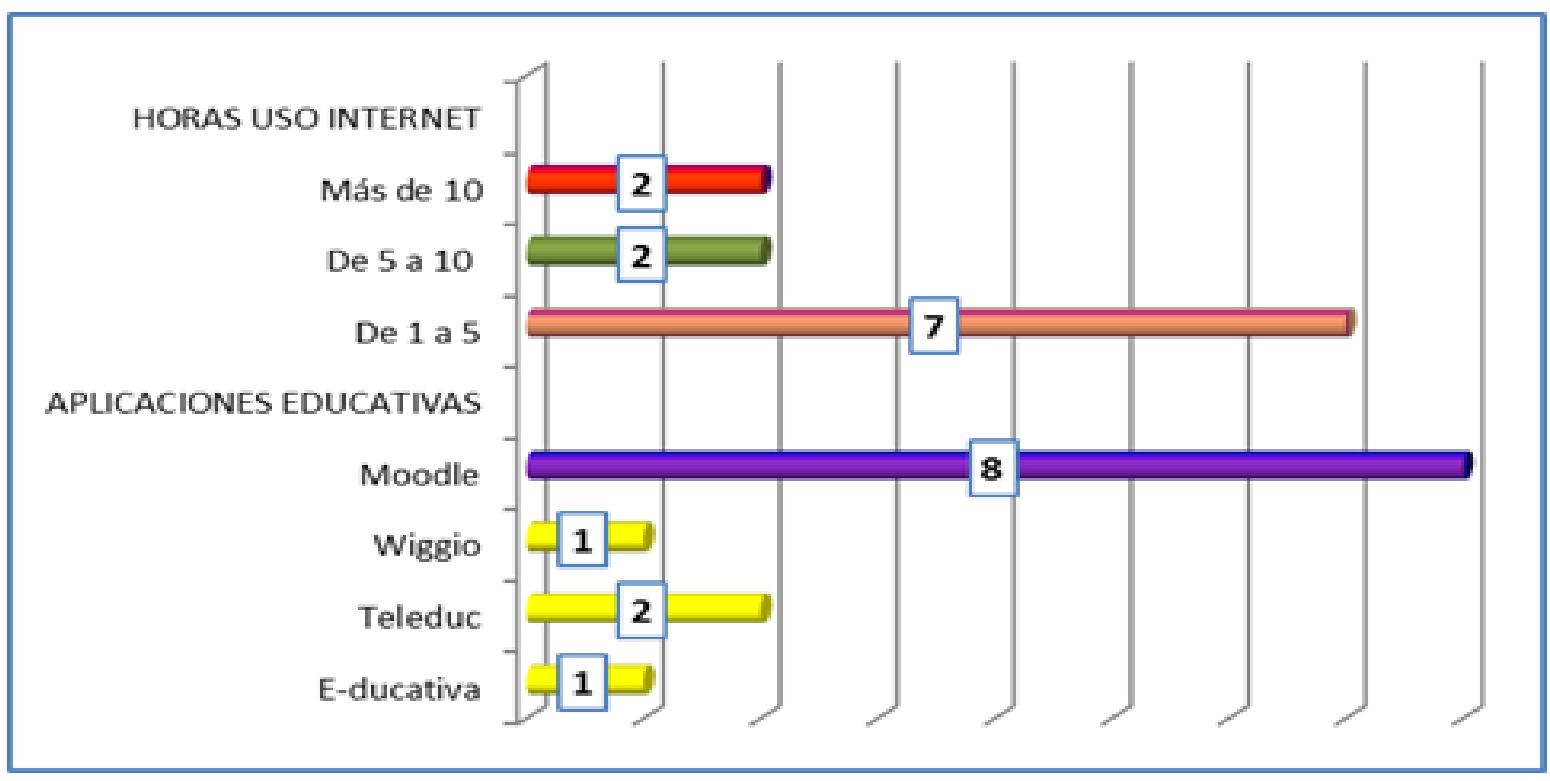

Revista Q | Vol. 10 | No. 20 | enero-junio 2016 


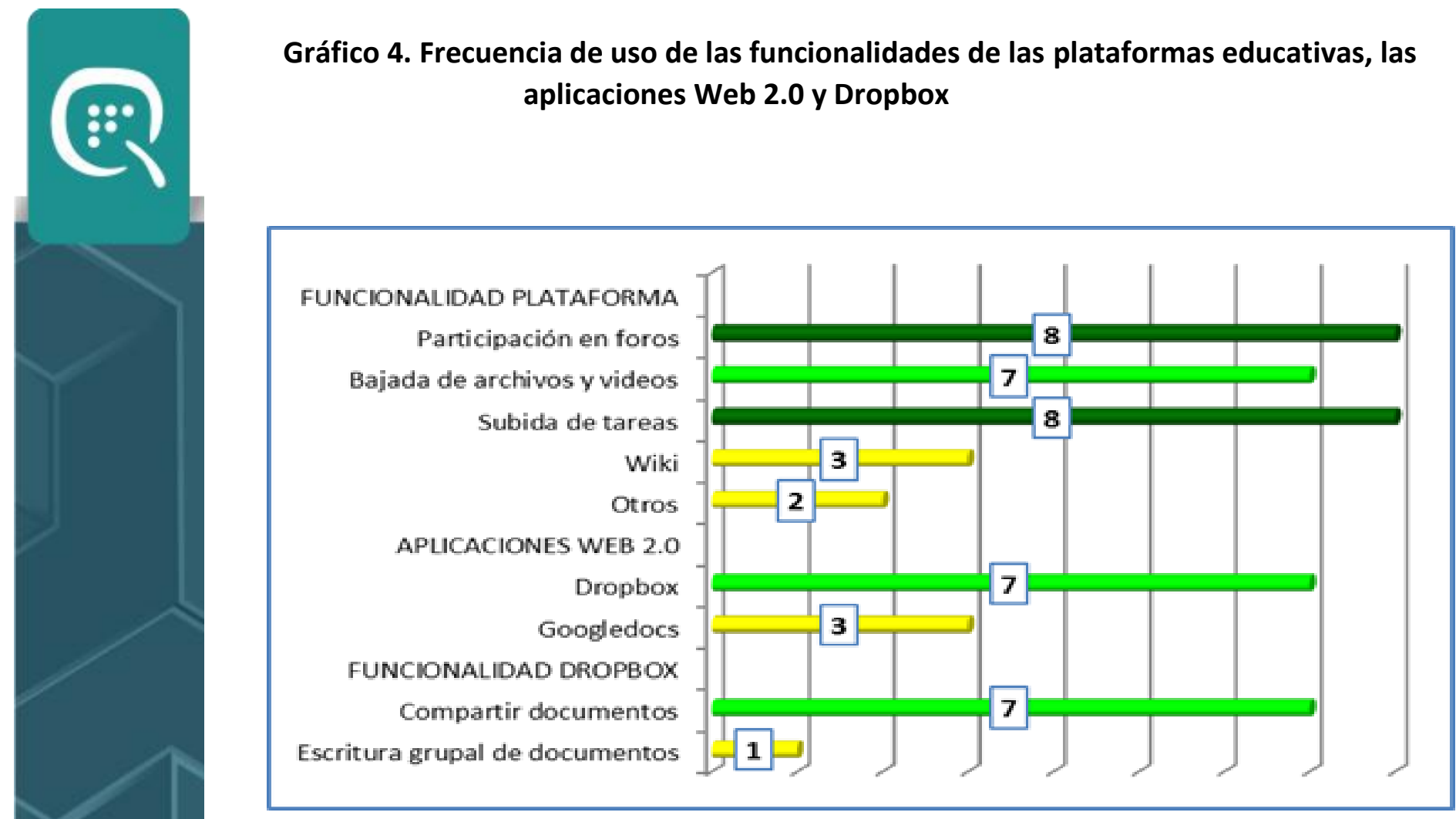

En síntesis, se advierte la máxima frecuencia de empleo $(\mathrm{N}=11)$ del procesador Word para la escritura básica de textos, la inserción de referencias, notas (al pie o al final), tablas, número

de página, pie de página y encabezado; un uso frecuente de inserción de citas y bibliografía, del diseño de página, de revisión de textos con control de cambios y de la inclusión de comentarios; los valores más bajos corresponden a inserción del índice y comparación de documentos, en ese orden. Por otra parte, la mayoría invierte de una a cinco horas diarias en Internet y emplea la plataforma Moodle para la participación en foros y la subida de tareas; medianamente alta (el 64\%) es la cantidad de estudiantes que la utilizan para la bajada de archivos y videos. También medianamente alto es el uso de Dropbox con el propósito de compartir documentos.

\section{Sobre la evaluación final}

Dado que el inventario de escritura académica se emplea en el diagnóstico (pre-test) y en la evaluación final (post-test), se grafican a continuación de modo comparativo los valores medios de ambas instancias: 


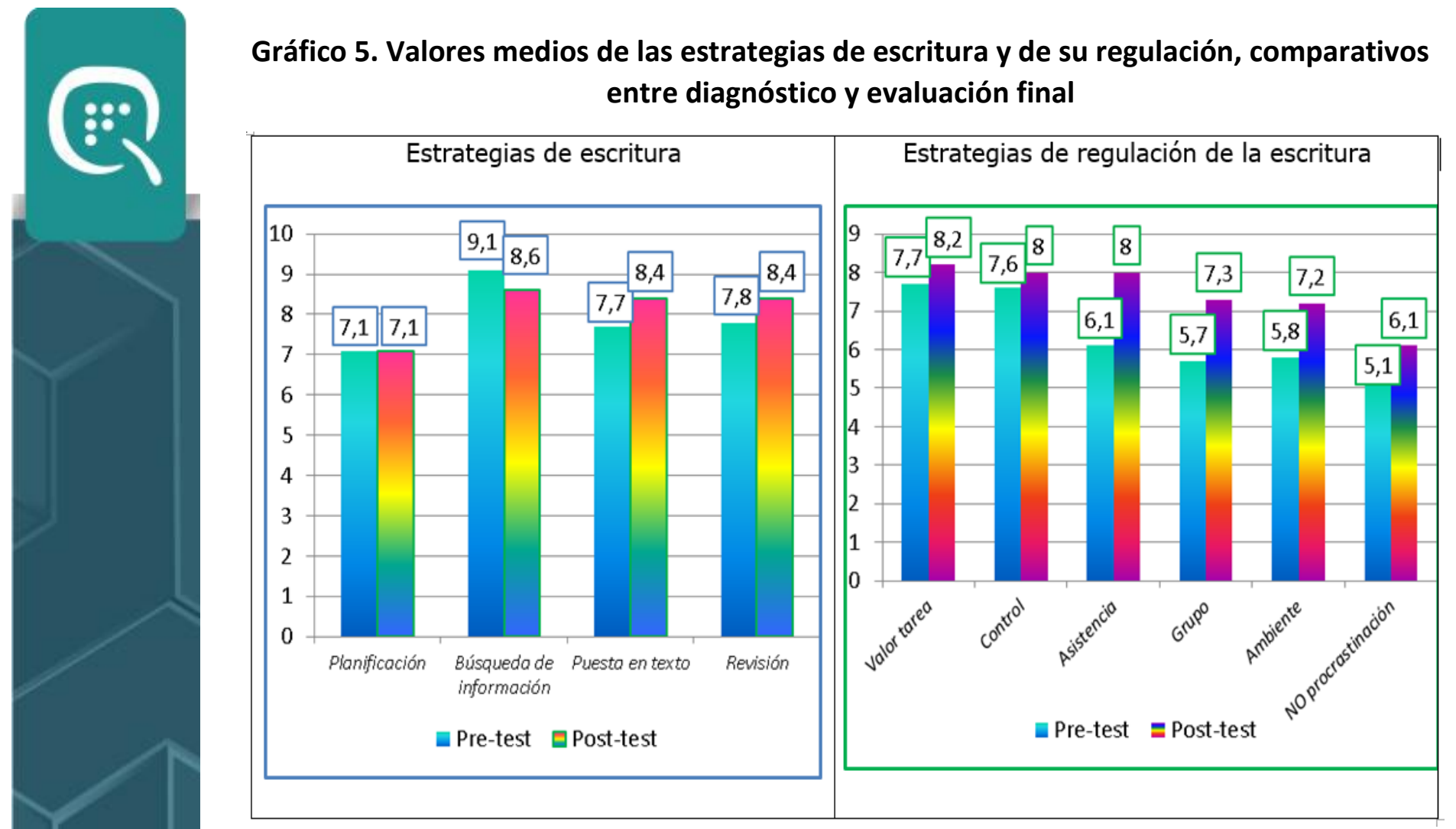

Gráfico 6. Valores medios de concepciones de escritura y de autoeficacia para la escritura, comparativos entre diagnóstico y evaluación final

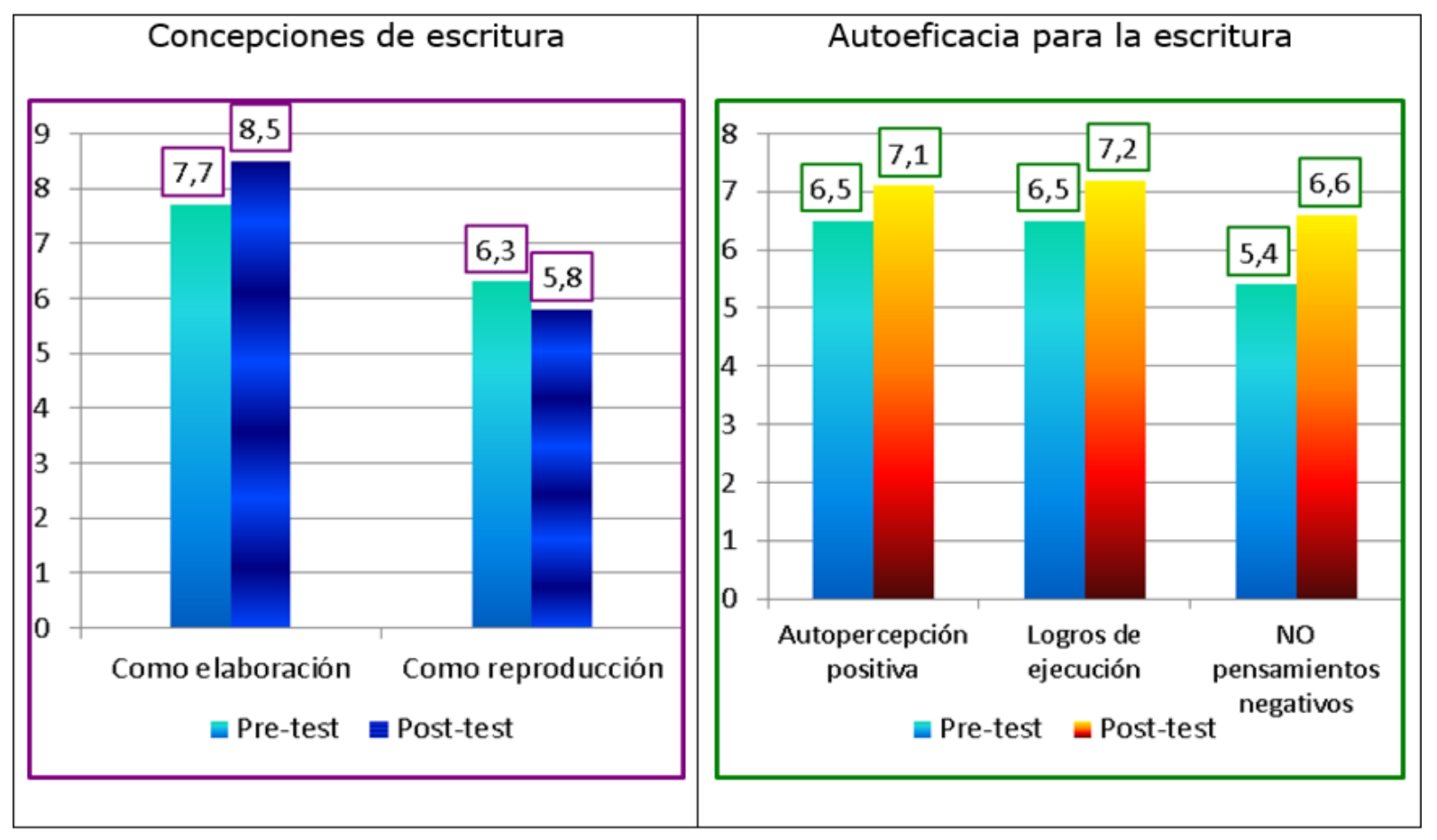

Revista Q | Vol. 10 | No. 20 | enero-junio 2016 


\section{Gráfico 7. Valores medios del compuesto "competencia autopercibida para la escritura académica" entre diagnóstico y evaluación final}

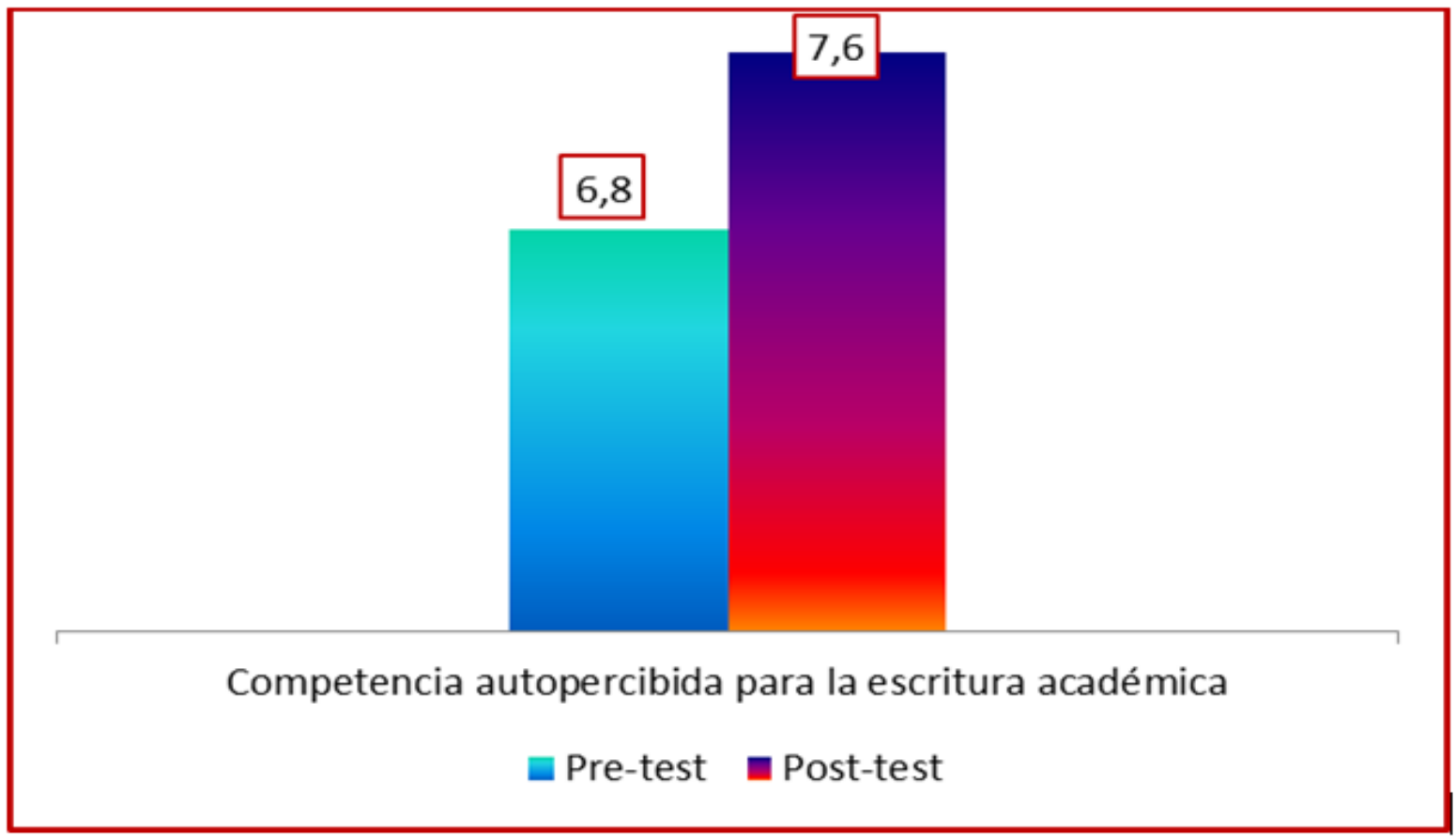

De estas divergencias sólo resultan estadísticamente significativas Búsqueda de asistencia (U de Mann-Whitney = 28,5; $p=0,03$ ), Apoyo del grupo ( $U$ de Mann-Whitney = 30,5; $p=0,04$ ) y No pensamientos negativos ( $U$ de Mann-Whitney $=32 ; p=0,05$ ). En cambio, si confrontamos categorías de desempeño (baja, media y alta) en cada dimensión de la variable, otra discrepancia alcanza significatividad estadística: Puesta en texto, una estrategia de escritura $\left(\mathrm{Chi}^{2}=10,444 ; \mathrm{gl}=2 ; \mathrm{p}=0,005\right)$. Finalmente, cuando se contrasta pre-test con post-test, siendo cada cursante su propio control (prueba de Wilcoxon), se hallan diferencias estadísticamente significativas en el incremento en dimensiones de la variable diferentes de las consignadas supra: Control $(z=2,265 ; p=0,02)$, Estructuración del ambiente y del tiempo $(z=2,149 ; p=$ $0,03)$, Escritura como elaboración $(z=2,671 ; p=0,008)$, Autopercepción positiva $(z=1,964 ; p$ $=0,05)$, Logros de ejecución $(z=2,100 ; p=0,036)$ y Competencia autopercibida para la escritura académica $(z=2,312 ; p=0,021)$.

En suma, la intervención pedagógica por intermedio de herramientas virtuales en estrategias para la producción conceptual y escrita en el doctorado, parece ejercer una incidencia positiva en distintos aspectos de la escritura, advirtiéndose dicho efecto con mayor claridad en los relativos a la regulación y a sentimientos de autoeficacia como escritor.

Respecto de las concepciones de investigación, se halla que las definiciones propias de investigación científica se distribuyen en tres categorías: 1 ) indagación analítica y sistemática ${ }^{7}$ (36\% de los cursantes), 2) creación de nuevo conocimiento 8 (45\%) y 3) resolución de un 


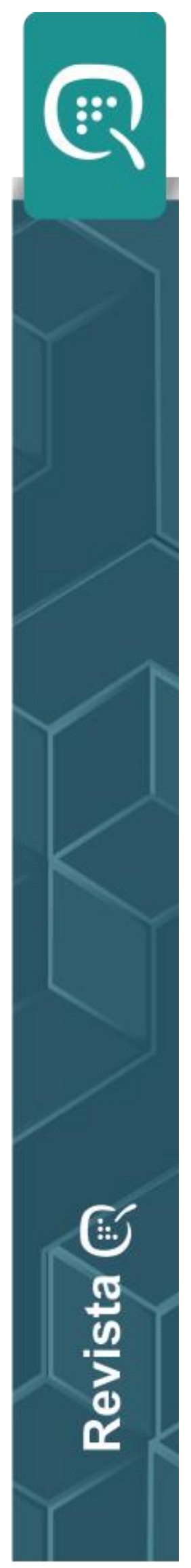

problema/reflexión sobre la práctica ${ }^{9}$ (18\%). De interés es que las concepciones de aprendizaje/conocimiento directa e interpretativa no evidencian asociación estadísticamente significativa con aquellas, mientras que sí lo hace la concepción constructiva ( $\mathrm{V}$ de Cramer = 0,$689 ; p=0,033$ ) ya que todos los doctorandos que conceptualizan la investigación como creación de nuevo conocimiento muestran puntajes medios en la concepción constructiva (y la mayoría, altos en interpretativa), al tiempo que los que adhieren a una conceptualización del trabajo científico como resolución de un problema/reflexión sobre la práctica alcanzan las puntuaciones superiores de la muestra en dicha concepción constructiva.

En relación con la pregunta “¿qué nociones sobre investigación científica has modificado a partir de tu experiencia de desarrollo de la tesis doctoral?", las respuestas se clasifican en cuatro categorías: 1 ) percepción del proceso ${ }^{10}$; 2 ) relativas al rigor metodológico ${ }^{11}$; 3 ) relativas al resultado que se espera ${ }^{12} ; 4$ ) otras (cualidades personales, función del encuadre teórico ${ }^{13}$ ); las tres primeras alcanzan el $27 \%$ y la última, el $18 \%$.

Mientras que la transformación nocional no se relaciona con los otros componentes analizados en las concepciones de investigación, sí lo hacen significativamente ( $\mathrm{V}$ de Cramer = 0,$735 ; p=0,018$ ) la conceptualización de las funciones del trabajo científico con las consideradas características de la buena investigación, como deja de manifiesto el análisis conjunto del sistema de concepciones que permite construir tres agrupaciones según rasgos compartidos:

- Grupo $1(\mathrm{~N}=4)$, que se caracteriza por la puntuación más alta en concepción directa, referir a cambios conceptuales relativos al rigor metodológico, considerar que una de las funciones de la investigación científica es "buscar apoyo empírico a una teoría o argumento" y que la buena investigación es "focalizada, rigurosa, metódica, bien diseñada y sistemática".

- Grupo $2(\mathrm{~N}=3)$, puntuación más alta en concepción interpretativa, definen la investigación como el proceso de creación de nuevo conocimiento, remiten a cambios vinculados con la percepción de <dicho proceso, entre las funciones del trabajo científico eligen "abordar aquellos temas que la disciplina respectiva considera importantes o urgentes" y juzgan que la buena investigación "es crítica del conocimiento existente, conceptualmente compleja y holística".

- Grupo $3(\mathrm{~N}=4)$, puntuación más alta en concepción constructiva; aunque eligen distintas funciones para la labor científica ("mejorar la situación problemática objeto de estudio", "constituir un aporte para sus destinatarios directos" u "orientarse hacia un logro valioso para la sociedad"), todas giran en derredor de optimizar la práctica y de allí que la buena investigación "es creativa e innovadora". 


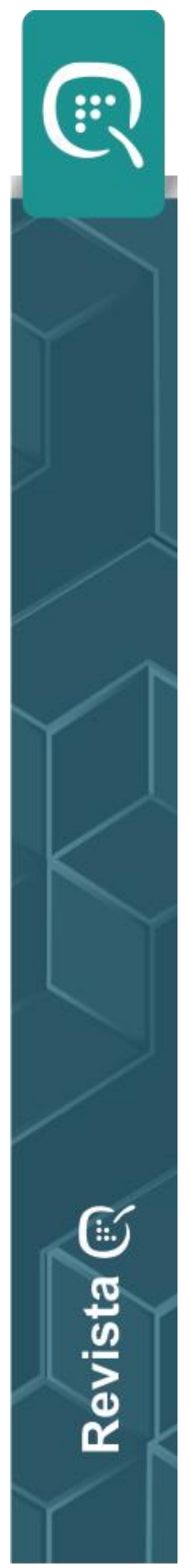

Los valores medios en las variables continuas según las agrupaciones que distinguiéramos se distribuyen como sigue:

Tabla 3. Puntajes de las variables continuas según las agrupaciones por concepciones de aprendizaje/conocimiento e investigación

\begin{tabular}{|c|c|c|c|c|c|c|c|c|c|c|c|c|c|}
\hline \multirow[b]{2}{*}{ Gr. } & \multirow[b]{2}{*}{ Cloze } & \multicolumn{2}{|c|}{ Estrategias } & \multicolumn{2}{|c|}{ Regulación } & \multicolumn{2}{|c|}{$\begin{array}{c}\text { E. como } \\
\text { Elaboración }\end{array}$} & \multicolumn{2}{|c|}{$\begin{array}{c}\text { E. como } \\
\text { Reproducción }\end{array}$} & \multicolumn{2}{|c|}{ Autoeficacia } & \multicolumn{2}{|c|}{$\begin{array}{l}\text { Competencia } \\
\text { autopercibida }\end{array}$} \\
\hline & & Pre & Post & Pre & Post & Pre & Post & Pre & Post & Pre & Post & Pre & Post \\
\hline 1 & 8,5 & 6,45 & 6,99 & 5,34 & 6,44 & 6,49 & 7,84 & 6,67 & 6,62 & 5,18 & 6,30 & 5,65 & 6,56 \\
\hline 2 & 9,2 & 8,71 & 8,89 & 6,54 & 7,75 & 8,56 & 8,94 & 5,88 & 5,00 & 6,35 & 7,43 & 7,16 & 8,03 \\
\hline 3 & 8,7 & 8,86 & 8,69 & 7,21 & 7,76 & 8,08 & 8,91 & 6,06 & 5,62 & 6,88 & 7,27 & 7,64 & 7,93 \\
\hline
\end{tabular}

La tabla muestra que el grupo 1, en correspondencia con la concepción directa, en el diagnóstico puntúa alto en la concepción de la escritura como reproducción y evidencia el puntaje inferior de la muestra en escritura como elaboración. En el grupo 2, prevalece esta última concepción, al tiempo que se halla el puntaje más bajo de la muestra en escritura como reproducción, tanto en el diagnóstico como en la evaluación final. El grupo 3 evidencia resultados similares al anterior, aunque con puntajes ligeramente inferiores en la primera variable y superiores en la segunda. En los tres grupos se advierte un incremento afín con el

nivel de partida, en consonancia con el conocido "efecto Matthew" (Stanovich, 1986) -con el tiempo, los que tienen un alto nivel de logro crecen con mayor rapidez que aquellos que parten de un nivel más bajo-, que resulta de mayor relevancia en: estrategias de regulación de la escritura, la concepción de la labor escritural como elaboración (esto es, como una empresa personal profunda y el compromiso con dicha conceptualización), sentimientos de autoeficacia como escritor y competencia autopercibida para la escritura académica.

El Cuestionario 3 de la evaluación final comprendió, como hemos adelantado, una serie de preguntas a partir de las cuales se solicitó a los estudiantes que indicaran las fortalezas y las limitaciones de las actividades (por un lado, las de realización individual y, por otro, las de resolución grupal) y de la propuesta del taller en general.

En relación con las actividades individuales, los alumnos indicaron dos aportes fundamentales. En primer lugar, consideraron que estas actividades promovieron la comprensión de diferentes dimensiones de las tesis como género, fundamentalmente de la situación comunicativa propia de la elaboración y la presentación, y de su organización lingüísticodiscursiva (i.e. secciones y movimientos de cada capítulo). En segundo lugar, consideraron que, a partir de los conocimientos adquiridos en las actividades resueltas individualmente, fue posible la revisión de la propia producción, en particular de la tesis de doctorado. En algunos 


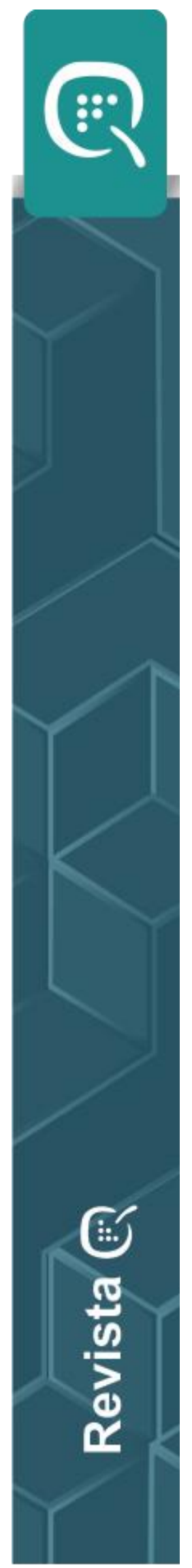

casos, los conocimientos adquiridos redundaron en las tareas de planificación de la escritura de la tesis; en otros, en el ajuste de capítulos ya elaborados. También se ha destacado la posibilidad de proyectar los conocimientos hacia el asesoramiento de tesistas. Las declaraciones de uno de los participantes del taller ponen en evidencia estos aportes:

\section{Ejemplo 1:}

"Me resultó muy útil tomarme el tiempo de leer sobre distintos aspectos y secciones de una tesis como objeto de estudio. He ordenado y ajustado ideas algo generales que tenía. Me ha resultado muy útil para mi propia tesis y para guiar más eficazmente a tesistas a quien ayudo."

En cuanto a las limitaciones de las actividades de resolución individual, se han señalado problemas con la organización del tiempo: incompatibilidades entre los tiempos relativos a la vida de los estudiantes, los relativos a la maduración cognitiva requerida por las actividades de análisis y producción textual, y los tiempos formales estipulados para la entrega de cada actividad. Por otra parte, los estudiantes sin formación de grado en Ciencias del Lenguaje han considerado que otra limitación importante ha sido la falta de conocimientos lingüísticos específicos para elaborar una reflexión metacognitiva sobre la producción textual, tanto la propia como la ajena. Veamos de qué manera expresan los mismos estudiantes estas dificultades:

\section{Ejemplo 2:}

"Mis problemas han tenido más que ver con mi deficiente manejo del tiempo."

\section{Ejemplo 3:}

"La mayor dificultad es mi falta de formación en habilidades lingüísticas de carácter gramatical."

Con respecto a las actividades de resolución grupal, los cursantes coincidieron en que el intercambio con colegas ha sido un aspecto muy enriquecedor y han destacado el valor de las interacciones para alcanzar acuerdos en torno a las actividades, tanto en relación con la distribución de los roles y las tareas, como del contenido en sí. Las interacciones, según los alumnos, han permitido conocer puntos de vista diferentes del propio en torno al contenido y a la forma de resolver la actividad, así como complementar esos puntos de vista. También se ha valorado el uso de tecnologías digitales específicas para el trabajo en grupo (i.e. Googledocs). Así, algunos estudiantes han señalado la posibilidad de poner en práctica dicha aplicación en futuras actividades académicas y profesionales. Las expresiones de una de las estudiantes permiten reconocer estas potencialidades de las actividades grupales:

\section{Ejemplo 4:}

“El trabajo grupal me ha permitido dialogar con otros acerca de cómo trabajar en producción de textos, tener lectores de lo que escribo y resolver situaciones de trabajo en equipo. 


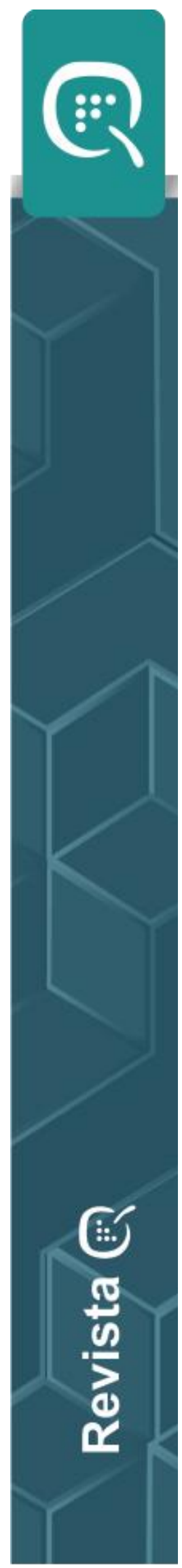

Además, fue una buena oportunidad para conocer la herramienta Google Docs, que me pareció sumamente útil, pues suelo hacer ponencias y artículos con una colega que no vive en la provincia."

Las limitaciones de las actividades grupales, como en el caso de las individuales, se han vinculado con el factor tiempo. Los estudiantes han destacado la dificultad para coordinar la disponibilidad de los miembros del grupo. Esto ha generado, en uno de los equipos, la falta de cumplimiento de los plazos de entrega. Asimismo, se ha manifestado que un problema importante de las actividades grupales ha estado representado por los niveles disímiles de participación de los estudiantes, con el consiguiente desequilibro en la distribución de las tareas o, en otros términos, la sobrecarga de trabajo de algunos integrantes por sobre otros.

\section{Ejemplo 5:}

"No todas podíamos en un mismo momento, o a veces resolvía más una que otras debido a la falta de comunicación."

Finalmente, las apreciaciones de los estudiantes permiten deducir que la valoración general de la propuesta del taller es positiva. Las limitaciones son puntuales y expresadas solo por algunos alumnos. El resto expresa explícitamente la falta de limitaciones, o se refiere a dificultades potenciales y explica de qué manera el taller las ha superado. Entre las principales fortalezas y aportes del taller, se destacan:

- Organización y conducción general del curso, y flexibilidad de los docentes, sobre todo con relación a los plazos de entrega.

- Acceso a bibliografía sobre escritura académica que resulta, para algunos estudiantes, muy novedosa y clarificadora.

- Desarrollo de contenidos que permiten la comprensión de diferentes dimensiones de la escritura académica y que, sobre todo, posibilitan la reflexión sobre la propia escritura de los estudiantes en instancias de posgrado.

Veamos, nuevamente, de qué manera se manifiestan estos aportes en la propia voz de los estudiantes.

\section{Ejemplo 6:}

"El taller fue idóneo para avanzar en la escritura de la tesis doctoral. Se proporcionaron los documentos necesarios y se desarrollaron actividades acordes con los objetivos propuestos. La metodología utilizada posibilitó la optimización de los tiempos, los recursos y las actividades programadas."

Las limitaciones indicadas, como hemos adelantado, han sido pocas y puntuales, pero significativas para optimizar el desarrollo de la propuesta. Por un lado, un estudiante ha referido la necesidad de organizar al menos un encuentro audiovisual con el grupo. En este 


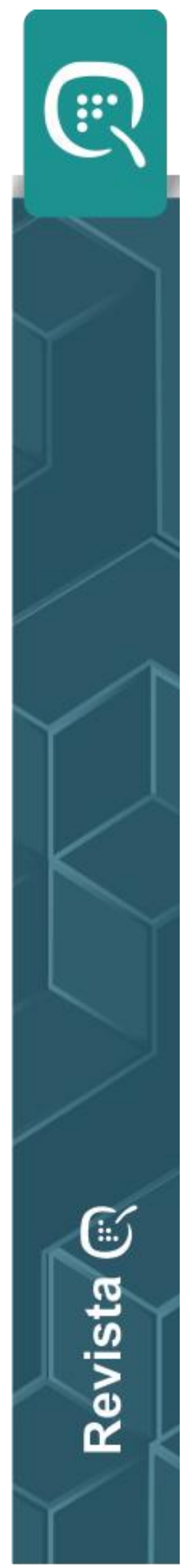

sentido, se han sugerido videoconferencias entre docentes y estudiantes agrupados por disponibilidades temporales. De esta manera, se aseguraría la intervención del grupo completo. Por otro lado, otra estudiante ha señalado el problema de algunas consignas de resolución grupal, que no aseguran que el trabajo sea realizado verdaderamente por todo el grupo. Se sugiere, en este sentido, revisar y ajustar algunas de las consignas de manera tal de propiciar una auténtica resolución colaborativa, y no simplemente una resolución que esté representada por la suma de subtareas resueltas individualmente.

\section{Conclusiones:}

En el presente artículo se comunican algunos hallazgos fundamentales de un primer ciclo de una investigación-acción, a partir de la cual, luego de un diagnóstico de las dificultades de los estudiantes para la escritura académica, se ha diseñado, implementado y evaluado un taller totalmente virtual orientado a promover la producción de un capítulo de la tesis de posgrado.

El ciclo de investigación-acción que se presenta en este trabajo parece mostrar, por un lado, una valoración positiva respecto de una propuesta de formación virtual como la desarrollada $y$, por otro, una incidencia positiva de la propuesta en el estímulo de dos "saltos cualitativos" necesarios para la producción de la tesis doctoral.

En relación con las valoraciones positivas sobre la propuesta, en general, y sus actividades, en particular, se destaca la posibilidad que ha brindado el curso de reflexionar sobre los rasgos genéricos de la tesis y, a partir de ello, revisar y ajustar la propia producción académica. En este sentido, parecería que los cursantes perciben que, como sugieren algunos estudios (Parodi, 1999, 2001), la identificación del contexto y la situación comunicativa de una tesis, del significado y contenido de la investigación, de la estructura canónica del texto, realizadas durante los procesos analíticos, contribuyen con la producción conceptual y escrita en el posgrado. Esto, a su vez, se vería favorecido por la interacción entre pares que permite complementar puntos de vista sobre los aspectos conceptuales y también sobre las modalidades de resolución de las tareas. Esta percepción coincide con los resultados de estudios que han implementado grupos formales o informales de escritura para contribuir con el mejoramiento de la producción académica (e.g. Aitchison y Lee, 2006; Lassig et al., 2009; Maher et al., 2008). El tiempo sería el factor que, por el contrario, obstaculizaría el trabajo. Los estudiantes han encontrado grandes dificultades para conciliar los tiempos propios, los tiempos madurativos de las actividades y los plazos de entrega, y este problema manifiesta nuevas aristas en las actividades grupales durante las cuales lo más complicado es compatibilizar los tiempos de diferentes cursantes. Las limitantes determinadas por el factor tiempo también han sido destacadas en otros estudios (Lassig et al., 2009), lo que nos enfrenta a la necesidad de diseñar estrategias que permitan optimizar y coordinar las disponibilidades temporales de diferentes alumnos. En este sentido, cabe destacar que la flexibilidad de los docentes involucrados ha sido identificada como una fortaleza de la propuesta. 


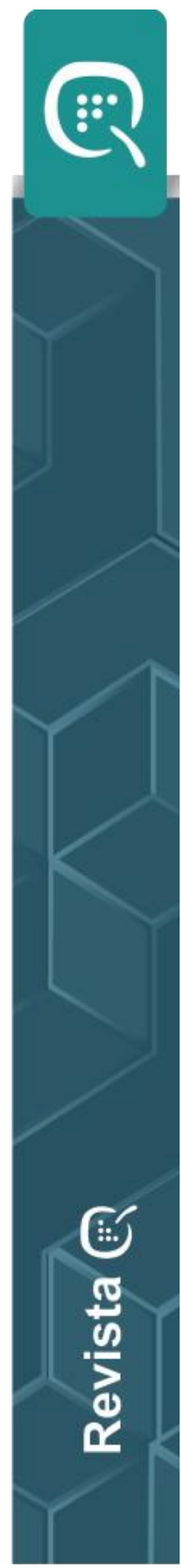

En cuanto a los dos "saltos cualitativos" que contribuyen con el ejercicio efectivo de la producción conceptual y escritura en el posgrado, el primero implica el tránsito desde una visón monolítica (el conocimiento como no problemático y que no necesita ser justificado) a una visión evaluadora del conocimiento y su formulación, tránsito que, respecto de la producción escrita, significa que el doctorando debe renunciar a un enfoque espontáneoimpulsivo o superficial -ausencia de planeamiento, selección de la información paso a paso sin una representación global del texto, una escritura reproductiva, que involucra una estrategia de listado, la inclusión de información de manera yuxtapuesta y acumulativa- y adoptar el enfoque que le permitirá escribir su tesis: un enfoque profundo, basado en adoptar una posición proactiva hacia la producción de un nuevo significado y el empleo de estrategias conducentes.

Un segundo salto cualitativo corresponde a una transición que se ha caracterizado como "crítica": el paso desde cursante de seminarios y consumidor de información, a investigador, a productor de un conocimiento original, con frecuencia a través de procesos inciertos en contextos poco estructurados. La elaboración de la tesis exige posicionarse como enunciador autorizado en una comunidad disciplinar de futuros pares (Carlino, 2005). A algunos doctorandos les cuesta transitar esta transformación; hay que ayudarlos a desarrollar un sentimiento de autoeficacia como escritor, lo que parece que el curso ha logrado andamiar.

De acuerdo con lo expuesto, es posible sugerir que un taller virtual para la enseñanza de la escritura de posgrado extendido en el tiempo puede expandir las opciones para la acción porque se formaliza como un ámbito con varias funciones interdependientes: el aprendizaje escritural en el marco de actividades genuinas de producción de la tesis, que promueven una posición proactiva hacia la generación de significados; la conformación progresiva de una comunidad discursiva, esto es, un espacio seguro para alcanzar acuerdos en torno a las actividades, compartir experiencias y escollos, a la vez que opera como grupo de apoyo y estímulo, garantizando cierta continuidad en las tareas; la formación "situada" en herramientas de investigación a propósito de situaciones problemáticas puntuales que cada grupo o participante debía resolver. En este sentido, el taller ha impulsado una actividad reflexiva sostenida tanto de parte de los alumnos, quienes valoraron el desafío de un trabajo metacognitivo intenso, como de parte de las tutoras para ir adecuando la propuesta a los emergentes, promover la participación, orientar y extender los análisis, señalar problemas, sistematizar y evaluar lo realizado. Como fruto importante de esta dinámica, el análisis ha sugerido que el taller representa un espacio efectivo para que los estudiantes, que son parte de instituciones académicas (e.g. docentes universitarios, becarios de universidades o centros de investigación) y en su mayoría se encuentran en etapas iniciales de la escritura de la tesis de posgrado, comiencen a transitar el pasaje de consumidores del conocimiento a miembros de una comunidad científica.

Para finalizar, consideramos que los resultados alcanzados permiten reconocer aspectos a los cuales dar continuidad, así como dimensiones que deben ser revisadas a fin de llevar a cabo un nuevo ciclo de diseño, implementación, evaluación y ajuste de la propuesta. Así, creemos 


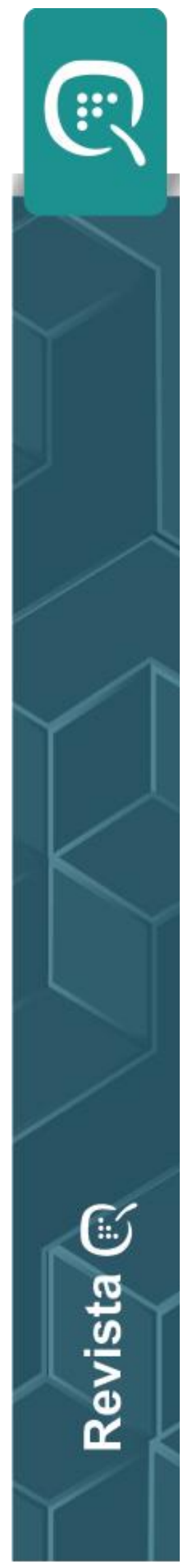

que los esfuerzos deben estar puestos en perfeccionar, por un lado, las formas de optimizar los tiempos de los estudiantes en las actividades y de coordinar efectivamente las disponibilidades en pro de desarrollos equitativos de los trabajos en equipo. Asumimos, entonces, la necesidad de encontrar estrategias para ayudar a los estudiantes a manejar y coordinar los tiempos -propios, ajenos y del taller- a fin de transitar de manera más efectiva la cursada. Creemos que las tecnologías digitales pueden ayudar a resolver esta problemática. Por otro lado, es necesario revisar algunas consignas de actividades grupales para que promuevan colaboraciones genuinas. Esta revisión debe ser realizada a la luz de las potencialidades que las tecnologías digitales han mostrado en prácticas de escritura grupal. Asumimos que en la interacción entre pares se encuentra una de las claves para favorecer una posición proactiva hacia la construcción de conocimiento original y hacia la transformación de estudiantes de posgrado en miembros de comunidades científico-académicas.

En definitiva, estamos convencidas de que las tecnologías digitales pueden contribuir a resolver las necesidades recién enunciadas. De hecho, la experiencia parecería mostrar un posible círculo hermenéutico entre tecnologías y educación (Davoli, Monari y Severinson Eklundh, 2009). Así, creemos que las futuras experiencias podrán confirmar en qué medida la enseñanza de la escritura en el posgrado requiere nuevas herramientas y aplicaciones para enseñar y aprender mejor, y, paralelamente, el uso de tecnologías requiere la adopción de nuevas y diferentes metodologías educativas. Nos interesa, particularmente, explorar cómo se da esta retroalimentación interpretativa entre tecnología y educación tanto en el plano teórico y conceptual como en el nivel de las prácticas concretas de enseñanza.

\section{Notas:}

1. Refiere a la obra de 1968.

2. El término cloze parece ser un neologismo, pues no figura en los diccionarios (Cairney, 1996, p. 95, nota del traductor).

3. El incremento de la muestra por sucesivas aplicaciones (entre 2012 y 2015) del instrumento original (en 100 ítems), ha llevado a recuperar ocho, por lo cual en esta ocasión el inventario cuenta con 78 ítems.

4. Constructo de Rothblum, Solomon y Murakami (1986) que refiere a la tendencia a posponer el trabajo, relacionada con el temor al fracaso; suele incluir: ansiedad generalizada, perfeccionismo, baja tolerancia a la frustración, incapacidad para pedir o aceptar ayuda, falta de autoconfianza, dificultad para tomar decisiones, una necesidad exagerada de estructura; se trata de una estrategia emocionalmente costosa y de autosabotaje.

5. 1) estrategias de escritura (que suma las cuatro dimensiones de la variable), 2) estrategias de regulación de la escritura (puntuación total de sus seis dimensiones), 3) concepciones de escritura (con dos variables: como elaboración y como reproducción), 4) autoeficacia 


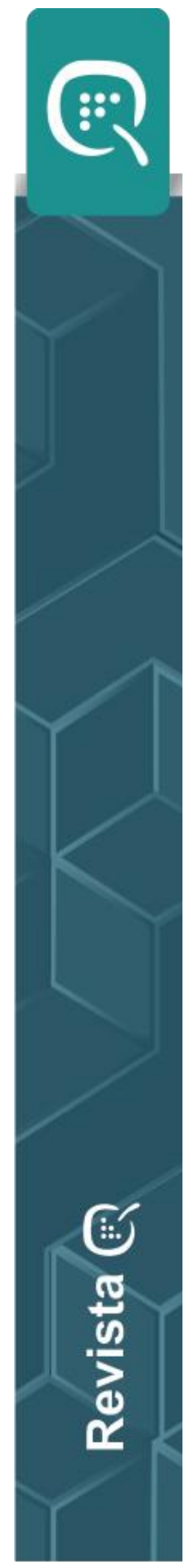

para la escritura (que adiciona sus tres dimensiones) y 5) el compuesto "competencia autopercibida para la escritura académica".

6. Desde los criterios de González Moreyra (1998) para la conversión de los puntajes del texto cloze en niveles funcionales de lectura, se halla que todos los participantes manifiestan nivel independiente (puntajes entre $75 \%$ y $100 \%$ ), esto es, leen con fluidez, precisión y comprenden la mayor parte del texto, subcategorizado en excelente ( $90 \%$ o más) que alcanzan tres alumnos y muy bueno (entre $75 \%$ y $89 \%$ ), categoría en la que se inscribe el resto de los cursantes (ocho alumnos).

7. Por ejemplo: "La investigación científica es un proceso riguroso, metódico, exhaustivo, sistemático y situado que posibilita construir, contextualizar y resolver problemas de investigación (...)" (doctoranda en Ciencias de la Educación, Universidad Nacional de Cuyo).

8. “Consiste en observar cuidadosamente un fenómeno desde un ángulo claramente delimitado e informado por una teoría rica y sugerente, para procurar formular generalizaciones válidas sobre el fenómeno. Alguna/s parte/s de estas generalizaciones resultarán, a su vez, aportes a la teoría que constituyó el horizonte teórico utilizado" (doctoranda en Letras, Universidad Nacional de Cuyo).

9. “Es el proceso por el cual se busca conocer en profundidad un fenómeno (en mi caso, social) para analizarlo desde la perspectiva de los actores involucrados a fin de sistematizar ideas y ofrecer nuevas miradas de intervención" (doctoranda en Ciencias de la Educación, Universidad de Buenos Aires).

10. "Lo que voy observando a medida que desarrollo mi tesis es que, mientras el horizonte especulativo se va ampliando, voy asiendo algunos mecanismos necesarios para cumplir con lo que se me pide, que no es perderme en el inmenso y enmarañado bosque de la bibliografía, sino deslindar los principales caminos recorridos y hacer el propio. (...)" (doctoranda en Letras, Universidad Nacional de Cuyo).

11. “(...) presto más atención a la forma sistemática en que debe desarrollarse una investigación, el entendimiento profundo del enfoque metodológico elegido para la disposición adecuada del material de estudio (...)" (doctoranda en Letras, Universidad Nacional de Cuyo).

12. “(...) proviniendo de una disciplina que se caracteriza por la 'creatividad' (en términos científicos, el método abductivo), durante el desarrollo de la tesis doctoral comprendí que hay mil formas de hacer una misma tesis, basta con cumplir las pautas o pasos científicamente consensuados, pero que solo una forma será la que ganará el 'Oscar'” (doctorando en Arquitectura, Universidad de Mendoza).

13. "La importancia de comprender cabalmente el estado del conocimiento, apoyarme sobre él y procurar avanzar en la comprensión de un fenómeno que me interesa conocer. Este avance implica una cuidadosa consideración y reconsideración de las observaciones que realizo. Según he avanzado en mi tesis, me ha resultado más y más importante la cuidadosa observación del objeto que analizo y la comprensión cabal de la teoría en la que me apoyo" (doctoranda en Letras, Universidad Nacional de Cuyo). 


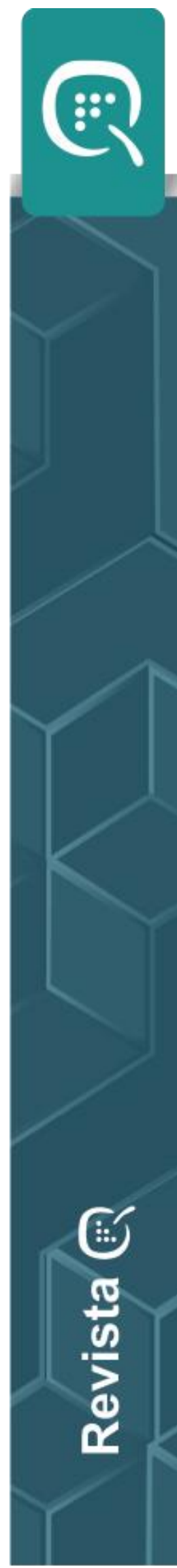

\section{Referencias}

Aitchison, C., y Lee, A. (2006). Research writing: problems and pedagogies. Teaching in Higher Education, 11(3), 265-278.

Álvarez, G. (2011). Principios para un final: reflexiones en torno a la escritura de la tesis de Maestría. Monográfico de la Maestría en Educación (pp. 43-53). Medellín: Universidad Pontificia Bolivariana.

Álvarez, G., y Bassa, L. (2013). TIC y aprendizaje colaborativo: el caso de un blog de aula para mejorar las habilidades de escritura de los estudiantes preuniversitarios. Revista de Universidad y Sociedad del Conocimiento (RUSC), 10(2), 5-19. Obtenido de http://rusc.uoc.edu/rusc/es/index.php/rusc/article/download/v10n2-alvarez-bassa/17405929-3-PB.pdf

Amezcua Prieto, C., Muñoz García, A., y Amezcua Membrilla, J.A. (2012). Variables motivacionales y teorías implícitas del aprendizaje en futuros profesores de Educación Secundaria. International Journal of Developmental and Educational Psychology, 1, 699-708. Obtenido de http://dehesa.unex.es/bitstream/handle/10662/2661

Arnoux, E., Borsinger de Montemayor, A., Carlino, P., Di Stefano, M., Pereira, C., y Silvestre, A. (2004). La intervención pedagógica en el proceso de escritura de tesis de postgrado. Revista de la Maestría en Salud Pública, 2(3), Publicación electrónica de la Maestría en Salud Pública de la Universidad de Buenos Aires. Obtenido de http://maestria.rec.uba.ar/Tres/articulos.htm

Backhoff Escudero, E., Larrazolo Reyna, N., y Rosas Morales, M. (2000). Nivel de dificultad y poder de discriminación del Examen de Habilidades y Conocimientos Básicos (EXHCOBA). Revista Electrónica de Investigación Educativa, 2(1). Obtenido de http://redie.uabc.mx/redie/article/view/15/26

Bereiter, C., y Scardamalia, M. (1987). The Psychology of Written Composition. Hillsdale, NJ: Erlbaum.

Caffarella, R. S., y Barnett, B. G. (2000). Teaching doctoral students to become scholarly writers: The importance of giving and receiving critiques. Studies in Higher Education, 25(1), 39-52. doi: 10.1080/030750700116000

Cairney, T. (1996). Enseñanza de la comprensión lectora. Madrid: Morata.

Campbell, J., Smith, D., y Brooker, R. (1998). From conception to performance: How undergraduate students conceptualize and construct essays. Higher Education, 36, 449-469. 


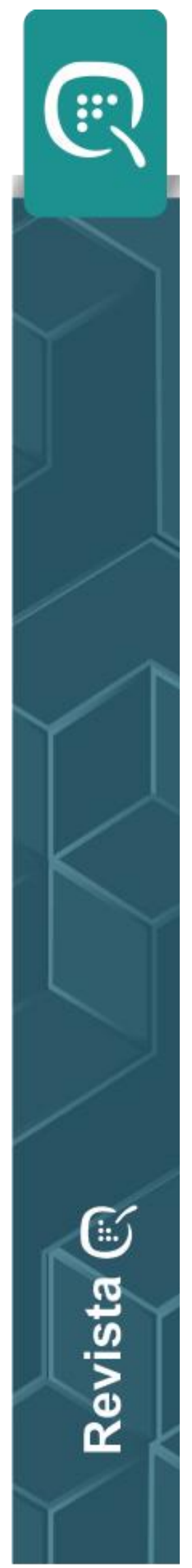

Carlino, P. (2005). La experiencia de escribir una tesis: contextos que la vuelven más difícil. Anales del Instituto de Lingüística, 24, 41-62. Obtenido de https://www.aacademica.org/paula.carlino/196.pdf

Cassany, D. (2000). De lo analógico a lo digital. El futuro de la enseñanza de la composición. Lectura y Vida, 21(4), 6-15. Obtenido de http://www.lecturayvida.fahce.unlp.edu.ar/numeros/a21n4/21_04_Cassany.pdf

Cubo de Severino, L., Puiatti, H., y Lacon, N. (Eds.) (2011). Escribir una tesis. Manual de estrategias de producción. Córdoba: Comunic-arte.

Dastjerdi, H., y Talebinezhad, M. (2006). Chain-preserving deletion procedure in cloze: a discoursal perspective. Language Testing, 23(1), 58-72.

Davoli, P., Monari, M., y Severinson Eklundh, K. (2009). Peer Activities on Web-Learning Platforms. Impact on Collaborative Writing and Usability Issues. Education and Information Technologies. 14(3), 229-254.

Difabio de Anglat, H. (2008). El test cloze en la evaluación de la comprensión del texto informativo de nivel universitario. RLA. Revista de Lingüística Teórica Aplicada, 46(1), 121-137. Obtenido de www.scielo.cl/pdf/rla/v46n1/art07.pdf

Difabio de Anglat, H. (2012). Hacia un inventario de escritura académica en el posgrado. Revista de Orientación Educacional, 26(49), 37-53.

di Stefano, M., y Pereira, C. (2004). La enseñanza de la lectura y la escritura en el nivel superior: Procesos, prácticas y representaciones sociales. En: Textos en contexto. Leer y escribir en la universidad (pp. 23-39). Buenos Aires: Asociación Internacional de Lectura.

Flower, L. (1979). Writer-based prose: A cognitive basis for problems in writing. College English, 41(1), 19-37. doi:10.2307/376357

Flower, L., y Hayes, J. (1996). Teoría de la redacción como proceso cognitivo. En: Textos en contexto (pp. 73-110). Buenos Aires: Asociación Internacional de Lectura.

González Moreyra, R. (1998). Comprensión lectora en estudiantes universitarios iniciales. Persona, 1, 43-65. Obtenido de http://fresno.ulima.edu.pe/sf\%5Csf_bdfde.nsf/imagenes/FCB815179CE767F105256F380056 E146/\%24file/gonzales.pdf 


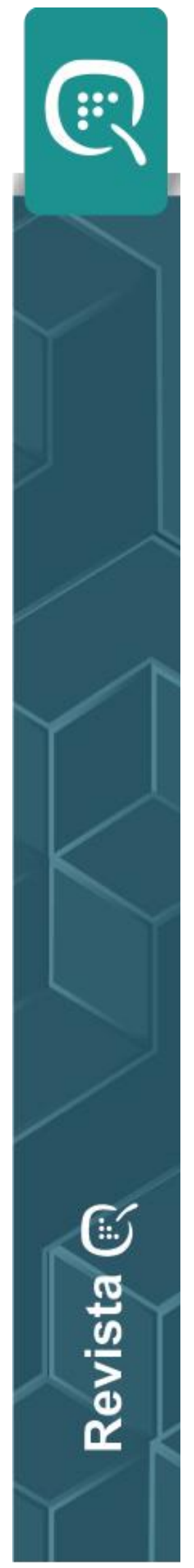

Hernández Sampieri, R., Fernández Collado, C., y Baptista Lucio, P. (2010). Metodología de la investigación (5a ed.). México: Mc Graw-Hill.

Hofer, B. (2001). Personal Epistemology Research: Implications for Learning and Teaching. Journal of Educational Psychology Review, 13(4), 353-383.

Hofer, B. (2004). Epistemological Understanding as a Metacognitive Process: Thinking Aloud During Online Searching. Educational Psychologist, 39(1), 43-55. Obtenido de

http://www4.ncsu.edu/ jilnietfe/Metacog_Articles_files/Hofer\%202004.pdf

Kletzien, S. (1991). Strategy use by good and poor comprehenders reading expository text of differing levels. Reading Research Quarterly, 26(1), 67-84.

Lassig, C., Lincoln, M., Dillon, L., Diezmann, C., Fox, J., y Neofa, Z. (2009). Writing together, learning together: the value and effectiveness of a research writing group for doctoral students. En: Australian Association for Research in Education 2009. International Education Research Conference, National Convention Centre, Canberra. Obtenido de http://eprints.qut.edu.au/28976/1/c28976.pdf

Lovitts, B. (2005). Being a good course-taker is not enough: a theoretical perspective on the transition to independent research. Studies in Higher Education, 30(2), 137-154. doi:

10.1080/03075070500043093

Maher, D., Seaton, L., Mcmullen, C., Fitzgerald, T., y Otsuji, E. A. (2008). Becoming and being writers: The experiences of doctoral students in writing groups. Studies in Continuing Education, 30(3), 263-275. doi: 10.1080/01580370802439870

Martínez-Fernández, J.R. (2004). Concepción de aprendizaje, metacognición y cambio conceptual en estudiantes universitarios de Psicología (Tesis de Doctorado). Universidad Autónoma de Barcelona. Barcelona. Obtenido de http://www.tdx.cat/handle/10803/2632

Martínez Fernández, J. R. (2007). Concepción de aprendizaje y estrategias metacognitivas en estudiantes universitarios de psicología. Anales de Psicología, 23(1), 7-16. Obtenido de http://www.redalyc.org/articulo.oa?id=16723102

Morchio, I., y Del Río Bayarri, A. (2015). Capítulo 2: Concepciones de aprender en alumnos universitarios de cinco carreras. En: I.L. Morchio (Coord.). Aprender a aprender como meta de la Educación Superior. Desde la comprensión de cómo aprende el alumno universitario a la promoción del aprendizaje autorregulado (pp. 81-120). Buenos Aires: Teseo. Obtenido de http://bdigital.uncu.edu.ar/objetos_digitales/8360/libro-aprenderaaprender-

comometaeducacionsuperior.pdf

Revista Q | Vol. 10 | No. 20 | enero-junio 2016 


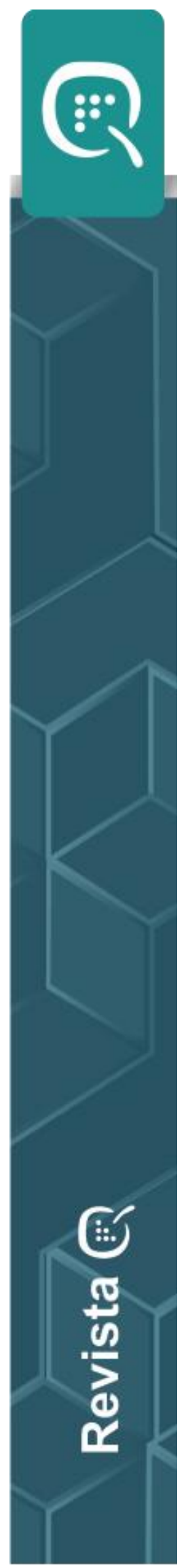

Parodi, G. (1999). Relaciones entre lectura y escritura: una perspectiva cognitiva discursiva. Valparaíso: Ediciones Universitarias de Valparaíso.

Parodi, G. (2001). Comprensión y producción lingüística: una nueva mirada al procesamiento del discurso escrito. Versión, 11, 59-97. Obtenido de https://www.researchgate.net/publication/277815945

Passig, D., y Schwartz, G. (2007). Collaborative Writing: Online versus Frontal. International Journal on e-Learning, 6(3), 395-412.

Perry, W. G., Jr. (1968). Patterns of development in thought and values of students in a liberal arts college: A validation of a scheme. Cambridge, MA: Bureau of Study Counsel, Harvard University.

Pozo, J. I., y Scheuer, N. (1999). Las concepciones sobre el aprendizaje como teorías implícitas. En: J. I. Pozo y C. Monereo (Coords.). El aprendizaje estratégico (pp. 87-108). Madrid: Santillana.

Rothblum, E., Solomon, L., y Murakami, J. (1986). Affective, cognitive, and behavioral differences between high and low procrastinators. Journal of Counselling Psychology, 33, 387394. Obtenido de http://rothblum.sdsu.edu/doc_pdf/procrastination/Affective_Cognative.pdf

Stanovich, K. E. (1986). Matthew effects in reading: Some consequences of individual differences in the acquisition of literacy. Reading Research Quarterly, 21, 360-407.

Vermunt, J. (1994). Inventory of Learning Styles in higher education: Scoring key. Tilburg University: Department of Educational Psychology.

Vermunt, J. (2005). Relations between student learning patterns, personal and contextual factors and academic performance. Higher Education, 49(3), 205-234. doi 10.1007/s10734004-6664-2

Zimmerman, B., y Risemberg, R. (1997). Becoming a Self-Regulated Writer: A Social Cognitive Perspective. Contemporary Educational Psychology, 22(1), 73-101. 